\title{
Molecular dynamics simulations of grain interactions in shock-compressed highly textured columnar nanocrystals
}

\author{
P. G. Heighway $\odot,{ }^{1, *}$ D. McGonegle, ${ }^{1}$ N. Park, ${ }^{2}$ A. Higginbotham, ${ }^{3}$ and J. S. Wark ${ }^{1}$ \\ ${ }^{1}$ Department of Physics, Clarendon Laboratory, University of Oxford, Parks Road, Oxford, OX1 3PU, United Kingdom \\ ${ }^{2}$ AWE, Aldermaston, Reading, Berkshire, RG7 4PR, United Kingdom \\ ${ }^{3}$ York Plasma Institute, University of York, York, YO10 5DD, United Kingdom
}

(Received 30 May 2019; published 19 August 2019)

\begin{abstract}
While experimental and computational studies abound demonstrating the diverse range of phenomena caused by grain interactions under quasistatic loading conditions, far less attention has been given to these interactions under the comparatively dramatic conditions of shock compression. The consideration of grain interactions is essential within the context of contemporary shock-compression experiments that exploit the distinctive $\mathrm{x}$-ray diffraction patterns of highly textured (and therefore strongly anisotropic) targets in order to interrogate local structural evolution. We present here a study of grain interaction effects in shock-compressed, body-centered cubic tantalum nanocrystals characterized by a columnar geometry and a strong fiber texture using large-scale molecular dynamics simulations. Our study reveals that contiguous grains deform cooperatively in directions perpendicular to the shock, driven by the gigapascal-scale stress gradients induced over their boundaries by the uniaxial compression, and in so doing are able to reach a state of reduced transverse shear stress. We compare the extent of this relaxation for two different columnar geometries (distinguished by their square or hexagonal crosssections), and quantify the attendant change in the transverse elastic strains. We further show that cooperative deformation is able to replace ordinary plastic deformation mechanisms at lower shock pressures, and, under certain conditions, activate new mechanisms at higher pressures.
\end{abstract}

DOI: 10.1103/PhysRevMaterials.3.083602

\section{INTRODUCTION}

When a polycrystal is compressed, the grains of which it is composed cannot deform as they would do in isolation, but must generally do so in such a way as to accommodate the presence of their neighbors [1]. This is to say that every grain must interact with those adjacent to it. Over the past several decades, extensive experimental and material modeling efforts have revealed a host of physical effects that can be directly attributed to interactions between grains, including changes to local orientation [2-10] and strain [10-14], generation of additional dislocations [15-18], and alteration of the macroscopic yield surface $[2,3,19,20]$. The vast majority of work to date has been performed in the context of quasistatic loading, where inertial forces between grains are negligible. By comparison, very little consideration has been given to the physics of grain interactions under the conditions of shock loading. Historically, progress in this area has been hampered by the fact that the highly transient shock state is inherently difficult to study experimentally: the typical shock-compression experiment provides a window of order nanoseconds in which to collect data, and the roster of diagnostic techniques capable of characterizing material behavior on such a timescale is severely limited. However, ultrafast $\mathrm{x}$ ray diffraction $(\mathrm{XRD})$ techniques have recently reached such a level of maturity that the evolution of lattice orientation $[21,22]$ and individual components of elastic strain [23-27] under shock conditions can now be resolved in situ with

*Corresponding author: patrick.heighway@physics.ox.ac.uk picosecond to femtosecond temporal resolution. The indirect observation of grain interactions via the changes they cause to local deformation is therefore, at least in principle, well within current experimental capabilities. We could thus be afforded the possibility of glimpsing the complex and dramatic grainscale dynamics taking place in the wake of the shock. Before attempting to realize this ambition, however, it is advisable to first gain an idea of what the effects of grain interactions are (and whether they are sufficiently large to be detected in experiment) with the aid of predictive material modeling.

The modeling of grain interactions under quasistatic loading conditions began in earnest just before the end of the 20th century. Prior to this time, the two extant species of polycrystal model, namely Taylor-type [28-32] and self-consistent models [19,33-35], were both incapable of faithfully treating interactions between grains; the former class could not guarantee that adjacent grains were in mechanical equilibrium, while the latter class could do so, but only in an average, mean-field sense. With rapid increases in readily available computing power, it became possible to move beyond these statistical models and seek numerical, full-field solutions, in which the stress and strain tensors are solved for on a discrete grid with subgrain resolution (with, e.g., the finite element method [36]) in such a way that grain interactions are automatically incorporated. The first studies capitalizing on these new numerical techniques quickly established that while statistical models could correctly predict the gross behavior of quasistatically deformed polycrystals, certain effects could only be explained with recourse to grain interactions. Such effects included: additional rotations [2,4], retrograde 
rotations $[4,20]$, and the formation of otherwise inexplicable textures [5]; the appearance of large intragranular orientation gradients, i.e., grain fragmentation [7,9,12,37]; and loss of correlation between each grain's initial orientation and its plastic strain state following loading $[11,12]$. Becker and Panchanadeeswaran [4] accounted for observations such as these by postulating two abstract "forces" that compete to control each grain's evolution: the first is intrinsic to the grain itself, and compels it to behave as it would do in isolation; the second is exerted upon the grain by its neighborhood, and forces it to undergo additional deformation to maintain compatibility and equilibrium with its neighbors at its boundaries. Subsequent statistical analyses suggested that, under certain circumstances, the latter force is actually dominant $[11,13]$. These computational studies have thus illustrated the significance of grain interaction effects, and although they were undertaken within the context of quasistatic loading, there is every reason to believe the same phenomena must manifest on some level in the shock regime.

In more recent years, a number of computational studies of multicrystals under shock-loading conditions have been undertaken with the aid of large-scale molecular dynamics (MD) simulations [38-46]. A small subset of these studies have focused on elucidating the physics of grain interactions, typically by modeling simple bicrystalline samples. These investigations have clearly demonstrated that during shock compression, grain interactions take on a dynamic nature due to the abruptness of the loading. Park [39] performed shockcompression simulations of a nickel bicrystal with its grain boundary normal perpendicular to the shock, and showed that when the shock front in one grain outpaces that in its neighbor, the former can laterally precompress the latter due to the transient stress gradients induced over the boundary within the elastic precursor. In a similar study of copper, Luo et al. [40] further showed that under high-symmetry conditions, lateral compression waves can coalesce at the center of the lagging grain, generating a moving "focus" at which the stress concentration is exceptionally high. One can speculate on the basis of investigations such as these that the ability of one grain to radically affect the stress state of its neighbors through dynamic interactions could have profound consequences for the manner in which each plastically deforms. The purpose of this paper is to build on these previous studies, and identify dynamic grain interaction effects in nanocrystals comprising more than two crystallites.

In this study, we concern ourselves with interactions in a particular class of body-centered cubic (bcc) polycrystal whose grains are characterized by (i) a columnar morphology and (ii) a fiberlike crystallographic texture, in which every grain has the same crystallographic direction closely aligned with its long axis (in this instance, [011]). Our reasons for doing so are twofold. First, the response of these polycrystals when shock-compressed along their fiber axis is relatively simple - the high aspect ratio of the crystallites means that interactions between grains take place almost exclusively in directions normal to the shock, while the fiber texture ensures the shock front progresses at approximately the same speed in every grain, meaning shock broadening effects are minimized. These polycrystals thus provide us with an uncomplicated environment in which to study the fundamentals of grain interactions. Second, tantalum targets with this morphological and crystallographic texture (which can be procured via vapor deposition) have already been successfully used to reveal the lattice-level dynamics that take place during shock compression [22,26] and subsequent release [27] using in situ XRD measurements. We further note that, as an axis of twofold rotational symmetry, the [011] fiber axis is particularly interesting from a grain-interaction point of view, because each grain will exhibit elastic and plastic anisotropy in directions normal to the shock. We have chosen to study bcc tantalum (Ta) here to provide better contact with the aforementioned experiments, but we stress that the fundamental grain interaction physics we shall illustrate is by no means peculiar to this element, as we will demonstrate.

The paper is structured as follows. In Sec. II, we explain in detail the methodology of our MD study, including descriptions of the structure of the simulated polycrystals, and the techniques used to characterize their plastic deformation and elastic strain state. The results of the simulations are detailed in Sec. III, which is sub-divided into three subsections. Section III A summarizes the response of single-crystal [011] Ta, in order to provide a reference point with which the behavior of grains in the polycrystals can be compared. In Sec. III B, we describe the behavior of the polycrystals compressed below their Hugoniot elastic limit (HEL), to better understand how grains interact without the added complication of crystal plasticity. We then detail the full elastoplastic response of the polycrystals to shock compression in Sec. III C, and highlight the behavior that is peculiar to the polycrystals, i.e., that which can be ascribed to grain interactions. A discussion of the results is given in Sec. IV, wherein we consider the implications of our study, and speculate on how it might be elaborated in further work. We then conclude in Sec. V.

\section{METHODOLOGY}

\section{A. Simulation method}

To study the response of nanocrystalline Ta to shock compression, we employ large-scale MD simulations using the LAMMPS code [48] and the embedded-atom model (EAM) Ta1 potential constructed by Ravelo et al. [49] This potential was tailored to reproduce the mechanical and thermodynamic properties of $\mathrm{Ta}$ at the megabar pressure scale. It successfully predicts the equation of state, elastic constants, and Hugoniot temperatures obtained from $a b$ initio calculations [49], as well as several experimentally determined high-pressure relations, including the pressure-volume curve at ambient temperature [50,51], the locus of shock states in the $u_{s}-u_{p}$ plane [52], and the melt curve [53].

The polycrystals we simulate comprise tessellating prismatic grains whose long axes are aligned with $z$, the direction along which the shock wave is launched. The bases of the prisms constitute the front and rear surfaces of the polycrystal, while the lateral faces make up the grain boundaries (GBs). We investigate two columnar geometries labeled the checkerboard (CB) and the honeycomb (HC), as depicted in Fig. 1. The former contains four grains with approximately square cross-sections, the latter six grains with hexagonal cross-sections. The minimum transverse dimensions of the 

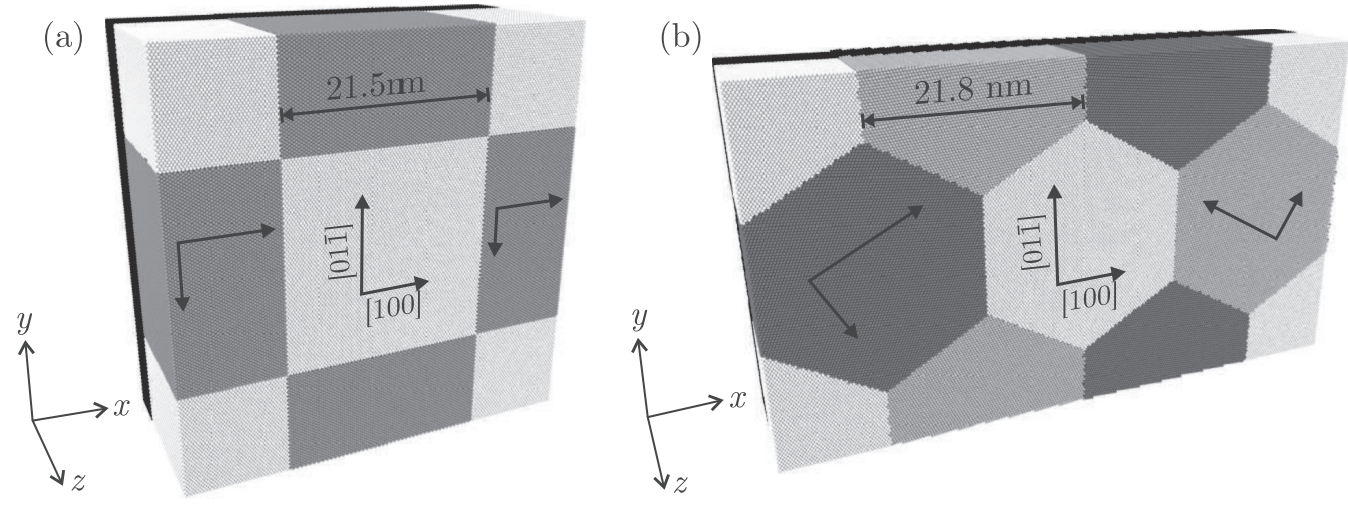

FIG. 1. Front end of the computational cells used in MD simulations of shock-compressed columnar polycrystals, visualized using OVITO [47]. (a) depicts the checkerboard (CB) geometry, (b) the honeycomb (HC). All grains have their [011] crystallographic axis aligned with $z$, the loading direction; the azimuthal orientation of each grain is indicated by the directions of the [100] and [011] axes. Grains with the same shading share the same orientation. The piston is depicted in black at the rear of the cells.

grains in the $\mathrm{CB}$ and $\mathrm{HC}$ geometries are 21.5 and $21.8 \mathrm{~nm}$, respectively. Both geometries span 1122 nm (i.e., 4800 [011] lattice spacings) in the compression direction. Periodic boundary conditions (PBCs) are applied to each polycrystal's $x z$ and $y z$ faces, while the $z$ direction is left aperiodic.

The orientations of the grains are chosen so as to emulate a fiber texture. In each geometry, there exists a central grain whose [100], [011] ], and [011] axes are aligned with the $x, y$, and $z$ directions, respectively. The orientations of the adjacent grains are related to that of the central grain by rotations about $z$. For the CB geometry, the misorientation angle is $90^{\circ}$. This angle is chosen to maximize the degree of anisotropy over the GBs, and exaggerate the magnitude of the stress gradients induced by shock-loading that cause the grains to interact. Our analysis is focused primarily on the CB geometry, though comparisons will be made with the less contrived $\mathrm{HC}$ geometry, for which the misorientation is only $60^{\circ}$. While the morphology and texture of these nanocrystals is highly idealized, they may serve as informative models, as they exhibit the basic physics relevant to a polycrystal in a way that is amenable to simple analysis.

To efficiently equilibrate the nanocrystals prior to loading, we exploit their translational symmetry along $z$ : rather than relaxing the crystals in their entirety, we first construct and relax a fully periodic supercell $9.4 \mathrm{~nm}$ in thickness, and subsequently replicate the cell 120 times in the $z$ direction to bring the system up to its full dimensions. The supercells are first simulated under the microcanonical (NVE) ensemble with a 300-K Langevin thermostat [54] to absorb the heat released from the GBs. Relaxation is then performed under isothermal-isobaric (NPT) conditions for 50 ps to anneal the GBs and bring the pressure to $0 \mathrm{GPa}$. Following the pressure correction, the system is time-integrated for a further $25 \mathrm{ps}$ under the canonical (NVT) ensemble (i.e., without pressure control); if the pressure has drifted by more than $1 \mathrm{kbar}$ following this interval, an additional NPT run is executed. This process is iterated until the pressure stabilizes.

The equilibrated supercell is then replicated in the compression direction, and the PBCs on the $x y$ faces of the simulation cell are relaxed. The full polycrystal is run under the NVE ensemble with a weak Langevin thermostat for
$50 \mathrm{ps}$ to damp out the pressure waves released from the front and rear surfaces. A final 50-ps period of relaxation without thermostatting concludes the equilibration. The polycrystals typically converge with residual pressures of order 10 bar and temperatures deviating from $300 \mathrm{~K}$ by no more than $0.1 \mathrm{~K}$. The only crystal defects present following relaxation are the GBs, whose excess energies in the $\mathrm{CB}$ and $\mathrm{HC}$ geometries are 1.35 and $1.23 \mathrm{~J} \mathrm{~m}^{-2}$, respectively.

In order to isolate the influence of one grain on another, we also perform MD simulations of single crystals with the same orientation as the central grain in the nanocrystals. By comparing the response of monocrystals and polycrystals subject to the same shock conditions, we can understand precisely how the stress and strain state of the grains is altered by virtue of their being situated in a polycrystal. The full dimensions of the single crystals are $24.8 \times 24.8 \times 1122 \mathrm{~nm}^{3}$. They are relaxed in the same manner as the polycrystals, and are also periodic in their transverse directions.

The single- and polycrystals are shock-loaded under the NVE ensemble with a 1-fs time step, using a piston instantaneously accelerated to constant velocity $\mathbf{u}_{p}=\left(0,0, u_{p}\right)$. Time integration proceeds until the compression wave reaches the end of the simulation cell. Note that the shock velocity $u_{s}$, and therefore the breakout time, is identical for every grain because each one has its [011] direction parallel to the loading axis.

\section{B. Plasticity mechanism identification}

To identify the deformation modes activated upon compression, we use a form of slip vector analysis (SVA), a technique originally formulated by Zimmerman et al. in the context of nanoindentation [55]. SVA allows one to locate slip planes, stacking faults, and twins by identifying atoms whose neighbors have suffered permanent displacement from their original sites in the unit cell due to the passage of a dislocation. The displacement vectors may be used to infer the Burgers vector of the dislocation responsible, while the plane(s) of motion may be determined by visualizing the structure formed by the slipped atoms. In brief, the algorithm we employ here first generates a "heat map" of the displacement 


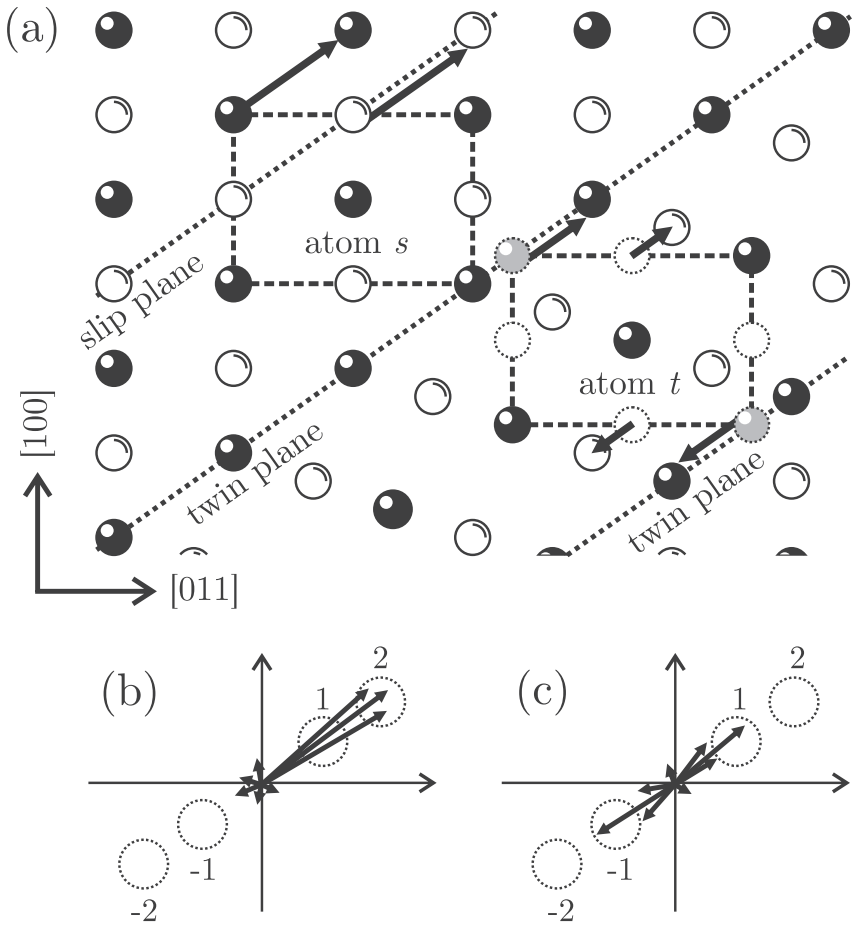

FIG. 2. Slip signatures for atoms in proximity to slip and twin events. (a) shows an ideal bcc crystal viewed along the [011] direction. Black and white atoms belong to separate (01) planes. Atom $s$ is located on a slip plane, atom $t$ in a twin. Arrows indicate the change in displacement of each atom's eight nearest neighbors following compression. (b) and (c) show these same changes (plus thermal noise) centered on a common origin for atoms $s$ and $t$, respectively. Each atom is characterized by the bins (labelled \pm 1 and \pm 2 ) into which their neighbor displacements fall.

vectors (whose maxima will be located at the Burgers vectors of the dislocations generated on compression), and then assigns atoms to slip or twin planes according to which of these maxima their particular neighbor displacements are found in proximity to. We now give a full description of the technique for completeness.

To illustrate the principle of SVA, we show in Fig. 2(a) a bcc crystal that has undergone plastic flow on nearby $(\overline{2} 11)$ planes. Two atoms are highlighted: atom $s$, which is located in proximity to a slip plane; and atom $t$, found in a twin. Each atom is distinguished by the particular combination of displacements of its eight nearest neighbors. For atom $s$, three neighbors are displaced by $1 / 2$ [111], while five suffer only thermal displacement. For atom $t$, two neighbors are displaced by $\pm 1 / 3$ [111], four by $\pm 1 / 6[111]$, and two by [000]. By exploiting these signatures, we can identify the slip or twin event in which the atom has participated.

In traditional SVA, the displacements of the central atom's neighbors are collapsed into a single metric called the slip vector $\mathbf{s}_{\alpha} \propto \sum_{\beta} \mathbf{u}_{\alpha \beta}$, where $\mathbf{u}_{\alpha \beta}$ is the displacement suffered by neighbor $\beta$ in the frame of $\alpha$, the central atom. While this step simplifies the resultant signature by reducing its dimensionality, it also renders the method unable to detect atoms located in deformation twins, for which $\mathbf{s}_{\alpha}$ vanishes. We circumvent this issue by retaining information about the individual displacements of each atom's neighbors, in such a way that slip and twinning can be treated equivalently. The version of SVA employed here is described below.

The first step is to establish the set of neighbor displacements frequently observed in the sampling region. Taking each atom as the central atom in turn, we calculate the change in relative position of the atoms with which it was coordinated before deformation. Let the trajectory of some atom $\gamma$ be denoted by $\mathbf{x}_{\gamma}(t)$. The change in displacement of neighbor $\beta$ from the central atom $\alpha$, as of time $t$, is given by

$$
\begin{aligned}
\mathbf{u}_{\alpha \beta} & =\left[\mathbf{x}_{\beta}(t)-\mathbf{x}_{\alpha}(t)\right]-\left[\mathbf{x}_{\beta}(0)-\mathbf{x}_{\alpha}(0)\right] \\
& =\mathbf{x}_{\alpha \beta}-\mathbf{X}_{\alpha \beta} .
\end{aligned}
$$

Here, the index $\beta$ runs over central atom $\alpha$ 's initial neighbors. We then generate a three-dimensional density plot of the set of all neighbor displacements $\left\{\mathbf{u}_{\alpha \beta}\right\}$. The displacements will cluster around a discrete set of points corresponding to the Burgers vectors of the dislocations generated on compression. We define a set of spherical bins located at these points with centers $\left\{\mathbf{b}_{i}\right\}$ and radii $\left\{r_{i}\right\}$. The finite radii of the bins account for both thermal noise and modulations in density and orientation. Since the density plot is necessarily centrosymmetric $\left(\mathbf{u}_{\alpha \beta}=-\mathbf{u}_{\beta \alpha}\right)$, bin $n$ is always implicitly paired with a second bin $-n$ located at $-\mathbf{b}_{n}$, as shown in Figs. 2(b) and 2(c). While it is not necessary to define the bins with the aid of a density plot if one has knowledge of the deformation modes a priori, doing so tends to increase the accuracy with which the atoms are classified.

The second step is to associate each atom with a plasticity mechanism according to the bins into which its neighbor displacements fall. Displacement $\mathbf{u}_{\alpha \beta}$ is said to belong to bin $n$ if $\left\|\mathbf{u}_{\alpha \beta}-\mathbf{b}_{n}\right\| \leqslant r_{n}$. If none of the bins are occupied, the central atom is assigned to slip event 0 , denoting an atom that has not undergone plastic flow. If only bins $\pm n$ are occupied, the atom is assigned to event $n$. If more than one type of bin is occupied, the atom is deemed unresolvable. To identify twinned atoms, for which more than one type of displacement exists, we ensure there are only bins located at either $\pm 1 / 3$ [111] or $\pm 1 / 6$ [111]; the former is preferable as the latter frequently overlaps the broad peak located at [000].

SVA has the advantage of being able to detect both dislocation slip and deformation twinning, but the technique suffers at high shock pressure. The greater the amount of plastic deformation a crystal has suffered, the greater the probability of its constituent atoms having participated in multiple plastic flow events. This effect causes the bins nearest the origin to empty out into the surrounding volume. Any atom located at the intersection between slip planes and twins can thus escape detection. In the following section, we describe a complementary algorithm with which we can detect deformation twins (but not slip planes) reliably at higher pressures.

\section{Elastic strain characterization}

The state of elastic deformation is one of several fundamental physical properties that is not explicitly evolved in MD simulations. The ability to extract elastic strain and rotation from MD, with either nearest-neighbor analyses [56] or by the study of synthetic diffraction data $[46,57,58]$, is vital if one wishes to compare its predictions with time-resolved $\mathrm{x}$-ray 
diffraction (XRD) experiments, in which the lattice $d$ spacings and texture of dynamically loaded matter can be measured in situ. Here, we describe the algorithm used to extract the local deformation state of an atomistic configuration, and subsequently to deconvolve the effects of lattice distortion and reorientation.

The algorithm may be summarized as follows. First, atoms located in noncrystalline environments are identified and rejected using common neighbor analysis (CNA) [59]. The algorithm then pairs the neighbors of each nondefective atom with ideal bcc neighbor vectors from one of a set of candidates bases (one representing the host lattice, the others any twins that could conceivably nucleate on compression). The linear operator that maps the ideal neighbor vectors onto the current neighbor vectors is then calculated, and subsequently re-expressed as a rotation followed by a series of elastic strains along the computational cell axes via a polar decomposition. The algorithm thus provides us with an elastic strain tensor for every nondefective atom, which can then be volume-averaged in order to calculate the elastic strain state of any given region of interest. We now go on to describe the full details of the calculation.

To characterize the local structure of the crystal, we employ the per-atom elastic deformation gradient $F_{\alpha}^{e}$. It may be defined for an atomistic configuration as the linear operator that maps each atom's neighbor vectors in an ambient, stress-free state onto its neighbor vectors following deformation, which we label $\left\{\mathbf{R}_{\alpha \beta}\right\}=\mathcal{R}$ and $\left\{\mathbf{x}_{\alpha \beta}\right\}$, respectively:

$$
F_{\alpha}^{e} \mathbf{R}_{\alpha \beta}=\mathbf{x}_{\alpha \beta} .
$$

In other words, if $\mathbf{x}_{\alpha \beta}$ is the vector joining atom $\alpha$ to neighboring atom $\beta$ after deformation has taken place, then $\mathbf{R}_{\alpha \beta}$ would be the displacement of atom $\beta$ from $\alpha$ if one relaxed all strains on the crystal. The means by which we calculate $\mathbf{R}_{\alpha \beta}$ for each neighboring atom will be explained shortly. To calculate $F_{\alpha}^{e}$, one requires at least three independent equations of the form above, each of which will provide three constraints on the nine elements of $F_{\alpha}^{e}$. For instance, if one knows the position of three neighboring atoms (labeled by indices $\beta=1,2,3$ ) and their corresponding ambient displacements, one can express three such vector equations simultaneously via the matrix equation

$$
F_{\alpha}^{e}\left(\begin{array}{lll}
\mathbf{R}_{\alpha 1} & \mathbf{R}_{\alpha 2} & \mathbf{R}_{\alpha 3}
\end{array}\right)=\left(\begin{array}{lll}
\mathbf{x}_{\alpha 1} & \mathbf{x}_{\alpha 2} & \mathbf{x}_{\alpha 3}
\end{array}\right),
$$

where $\left(\begin{array}{lll}\mathbf{u} & \mathbf{v} & \mathbf{w}\end{array}\right)$ is the $3 \times 3$ matrix whose columns are the vectors $\mathbf{u}, \mathbf{v}$, and $\mathbf{w}$. This equation is sufficient to uniquely determine $F_{\alpha}^{e}$ :

$$
F_{\alpha}^{e}=\left(\begin{array}{llll}
\mathbf{x}_{\alpha 1} & \mathbf{x}_{\alpha 2} & \mathbf{x}_{\alpha 3}
\end{array}\right)\left(\begin{array}{lll}
\mathbf{R}_{\alpha 1} & \mathbf{R}_{\alpha 2} & \mathbf{R}_{\alpha 3}
\end{array}\right)^{-1} .
$$

Depending on which of atom $\alpha$ 's neighbors one uses in Eq. (4), one may obtain slightly different results due to perturbative effects like thermal noise. To mitigate such effects, we take $F_{\alpha}^{e}$ to be the arithmetic mean of all possible elastic deformation gradients calculable from $\alpha$ 's 14 nearest and next-nearest neighbors (of which 232 exist). From $F_{\alpha}^{e}$, it is then possible to infer the extent of both the distortion and reorientation of atom $\alpha$ 's unit cell.

The elastic deformation gradient can only be meaningfully defined for atoms that retain a crystalline environment following shock loading. It is therefore desirable to exclude atoms located in proximity to crystal defects from the computation. Here, we use adaptive common-neighbor analysis (a-CNA), as defined by Stukowski [60], to identify and reject noncrystalline material before calculation of $F_{\alpha}^{e}$ begins. a-CNA is a simple extension of the traditional CNA technique [59] where the maximum separation of bonded atoms, or cutoff radius $r^{\text {cut }}$, is calculated for each atom individually. One first infers the local lattice constant $a_{\alpha}$ using the displacements of the atoms nearest $\alpha$. The expression appropriate for a bcc crystal reads

$$
a_{\alpha}=\frac{1}{14}\left(\sum_{\beta=1}^{8} \frac{2}{\sqrt{3}}\left\|\mathbf{x}_{\alpha \beta}\right\|+\sum_{\beta=9}^{14}\left\|\mathbf{x}_{\alpha \beta}\right\|\right) .
$$

The first sum runs over the eight nearest neighbors in the first coordination shell (displaced on average by $\|1 / 2\langle 111\rangle\|=$ $\left.\sqrt{3} / 2 a_{\alpha}\right)$, while the second sum accounts for the six nextnearest neighbors in the second coordination shell (displaced by $\left.\|\langle 100\rangle\|=a_{\alpha}\right)$. The cutoff is then placed between the second and third shells, i.e., $r_{\alpha}^{\text {cut }}=1 / 2(1+\sqrt{2}) a_{\alpha}$. By dynamically calculating the cutoff in this way, we are able to filter shock-compressed crystals without the need to manually select the cutoff radius to suit the local density: the broad range of compression states accessible to the dynamically loaded material often cannot be satisfactorily characterized by a single, static cutoff. We note that this technique is known to fail at temperatures approaching the (pressure-dependent) melting temperature $T_{m}$, where CNA frequently misidentifies crystalline material as defective due to sporadic breaking of bonds [61]. However, these false negatives are not expected to appreciably influence our statistics, as our study only concerns Hugoniot states for which $T<0.35 T_{m}$.

To calculate $F_{\alpha}^{e}$, one must have a consistent means of assigning to each of $\alpha$ 's current neighbors an ideal neighbor vector, i.e., of assigning to each $\mathbf{x}_{\alpha \beta}$ an $\mathbf{R}_{\alpha \beta}$. The mapping should fulfill the conditions of local compatibility [62]: for any three neighboring atoms $\alpha, \beta$, and $\gamma$ in a nondefective region of the crystal, the assignments must be such that $\mathbf{R}_{\alpha \beta}+\mathbf{R}_{\beta \alpha}=0$ and $\mathbf{R}_{\alpha \beta}+\mathbf{R}_{\beta \gamma}+\mathbf{R}_{\gamma \alpha}=0$. For instance, if when treating atom $\alpha$ the algorithm determines that atom $\beta$ is displaced from $\alpha$ by [100], it must when treating atom $\beta$ assert that atom $\alpha$ is displaced from $\beta$ by [100]. These conditions essentially guarantee that the nominal orientation of adjacent unit cells is consistent, so the deformation gradient varies quasi-continuously throughout each grain and twin. The mapping method we use here is not unlike that detailed in Ref. [63], wherein the neighbor displacement vectors $\left\{\mathbf{x}_{\alpha \beta}\right\}$ are independently matched to ideal vectors in a template bcc structure according to their similarity. The implementation of this technique, depicted schematically in Fig. 3, is described below for completeness.

We first specify the set of 14 ambient neighbor vectors $\left\{\mathbf{B}_{i}^{(0)}\right\}=\mathcal{B}_{0}$ that describe the first and second bec coordination shells for the host orientation. In anticipation of the onset of deformation twinning, we also construct $M$ further twin bases $\mathcal{B}_{1,2, \ldots, M}$. The elements of each twin basis are constructed by reflecting the elements of $\mathcal{B}_{0}$ in the corresponding twin plane with unit normal $\hat{\mathbf{m}}$ :

$$
\mathbf{B}_{i}^{(m)}=\mathbf{B}_{i}^{(0)}-2\left(\mathbf{B}_{i}^{(0)} \cdot \hat{\mathbf{m}}\right) \hat{\mathbf{m}} .
$$



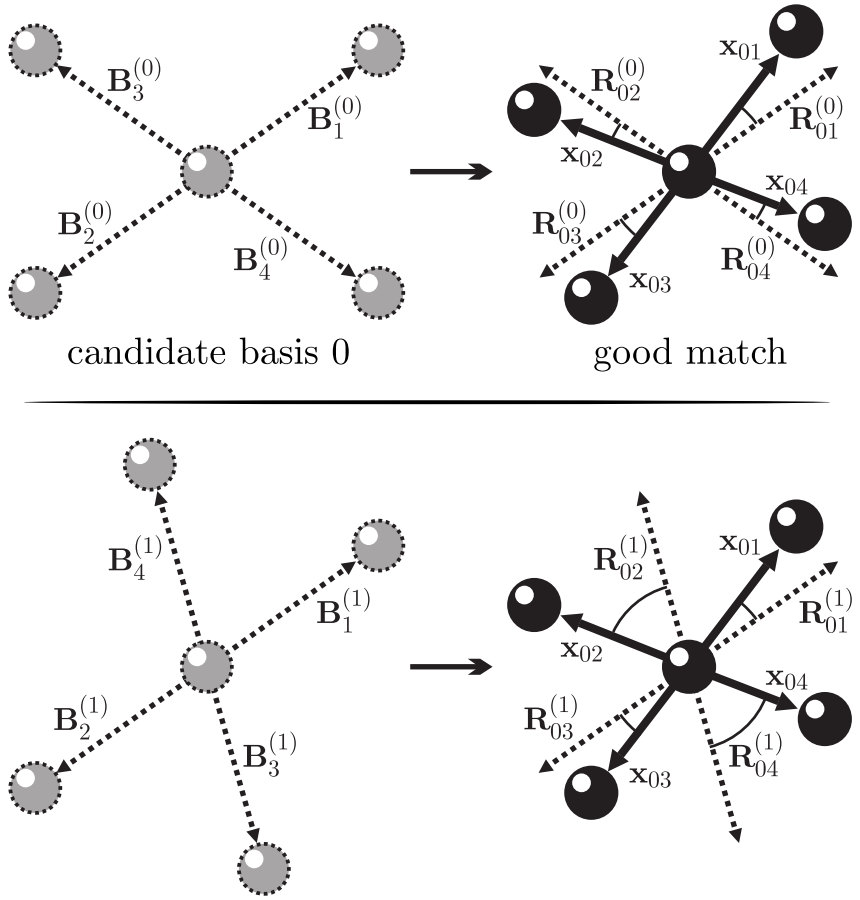

candidate basis 1

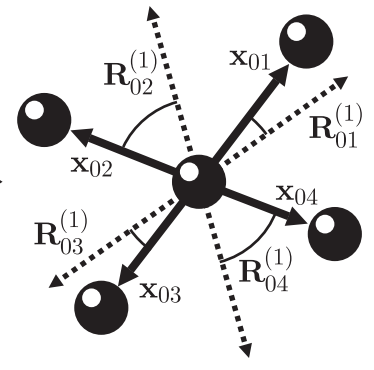

poor match

FIG. 3. Schematic depicting the mapping stage of the elastic strain calculation algorithm. (Left) Two candidate bases representing the host and twin orientations with associated ideal neighbor vectors $\left\{\mathbf{B}_{i}^{(0)}\right\}$ and $\left\{\mathbf{B}_{i}^{(1)}\right\}$, respectively. (Right) Pairing-off of the ideal neighbor vectors and the current neighbor displacements $\left\{\mathbf{x}_{0 \beta}\right\}$ according to cosine similarity for central atom 0 . In this case, basis 0 would be deemed more consistent because the total angle subtended by its paired vectors is smaller.

A separate set of $M+1$ candidate bases must be constructed for each grain under consideration. Then, taking each basis in turn, we assign each of the central atom's 14 nearest and next-nearest neighbors to an ideal neighbor vector. Vector $\mathbf{x}_{\alpha \beta}$ is paired with the ideal vector with which it subtends the smallest angle, or equivalently, the vector with which it has maximal cosine similarity. Formally expressed, this reads

$$
\mathbf{R}_{\alpha \beta}^{(m)}=\underset{\mathbf{B}_{i}^{(m)} \in \mathcal{B}_{m}}{\arg \max } \operatorname{sim}\left(\mathbf{x}_{\alpha \beta}, \mathbf{B}_{i}^{(m)}\right),
$$

where

$$
\operatorname{sim}(\mathbf{u}, \mathbf{v})=\frac{\mathbf{u} \cdot \mathbf{v}}{\|\mathbf{u}\|\|\mathbf{v}\|}
$$

We then determine the basis that best describes the local unit cell of $\alpha$. The basis identified with $\mathcal{R}$ is the one that maximizes the total cosine similarity $\sum_{\beta=1}^{14} \operatorname{sim}\left(\mathbf{x}_{\alpha \beta}, \mathbf{R}_{\alpha \beta}^{(m)}\right)$. If the pairing of current and ideal neighbor vectors is not one-to-one for a given basis, that basis is excluded from consideration. If none of the bases provide one-to-one mappings, the atom is deemed unresolvable and is excluded from the computation.

The mapping method above is generally successful, though there do exist isolated instances of local compatibility not being satisfied: small clusters occasionally form whose orientation is "equidistant" between two or more valid but mutually inconsistent mappings. An atom in such a cluster will vacillate between these different mappings as thermal noise perturbs

the displacement of its neighbors. These errors occur whenever the lattice orientation is not sufficiently well-matched to any one of the candidate basis orientations. One could in principle correct the errors by recursively adjusting the bond mappings of each atom to match those of its neighbors, as is done in the dislocation extraction algorithm (DXA) [62,64]. However, the fraction of the entire crystal these incompatible atoms constitute is typically less than $10^{-4}$, and therefore spatial averages of the local deformation state are not expected to be adversely affected.

Note that this mapping method also provides us with a second and more robust means of identifying deformation twins, independent of the SVA technique described in Sec. IIB. However, it is of course incapable of detecting slip events, which leave in their wake a pristine crystal with the host orientation.

The elastic deformation gradient encodes information about both the distortion and reorientation of the unit cell. The final step in the algorithm is to separate these two effects by calculating a polar decomposition of $F_{\alpha}^{e}$. This is a re-expression of the deformation as a strain followed by a rotation, or vice versa. The decomposition we use reads

$$
F_{\alpha}^{e}=V_{\alpha}^{e} R_{\alpha},
$$

where $R_{\alpha}$ is the rotation matrix, and the symmetric matrix $V_{\alpha}^{e}$ is the left stretch tensor. The (engineering) elastic strain in the computational cell basis, $\varepsilon_{\alpha}^{e}$, is related trivially to the stretch tensor by $\varepsilon_{\alpha}^{e}=V_{\alpha}^{e}-I$. We will henceforth drop the superscript $e$, as the elastic strain is the only type of strain to which we will make explicit reference.

\section{RESULTS}

\section{A. Single-crystal response}

It is instructive to first establish how [011] Ta responds to shock compression at the level of a single grain, that is, in the absence of polycrystalline effects. To do so, we consider the behavior of monocrystals shock-compressed to at least $40 \mathrm{GPa}$ (the HEL for a crystal modeled under the Ta1 potential shocked along [011] [49]) that are periodic in directions normal to the shock. These monocrystals serve as the "control group" with which we will eventually compare polycrystals loaded under similar shock conditions in order to isolate the additional physics introduced by intergranular interactions. We are primarily interested in establishing whether the response of these [011] single crystals is (given their low rotational symmetry about $z$ ) markedly different in the two directions normal to the shock, both during the plastic relaxation phase and in the steady state that follows. As we shall show in Sec. III B, a grain that exhibits an anisotropy of this sort is bound to interact with its neighbors.

We first identify the plasticity mechanisms activated in [011] Ta under shock compression by means of the SVA method described in Sec. II B. In Figs. 4(a) and 4(b), we show cross-sections of the neighbor-displacement density plot in the $x z$ and $x y$ planes obtained from a single crystal shocked to $40 \mathrm{GPa}$. Atoms whose neighbor displacements are found in proximity to labeled peaks in the density plot are shown in Figs. 4(c) and 4(d) for representative regions of the crystal. The SVA reveals that plastic deformation occurs in two stages. 

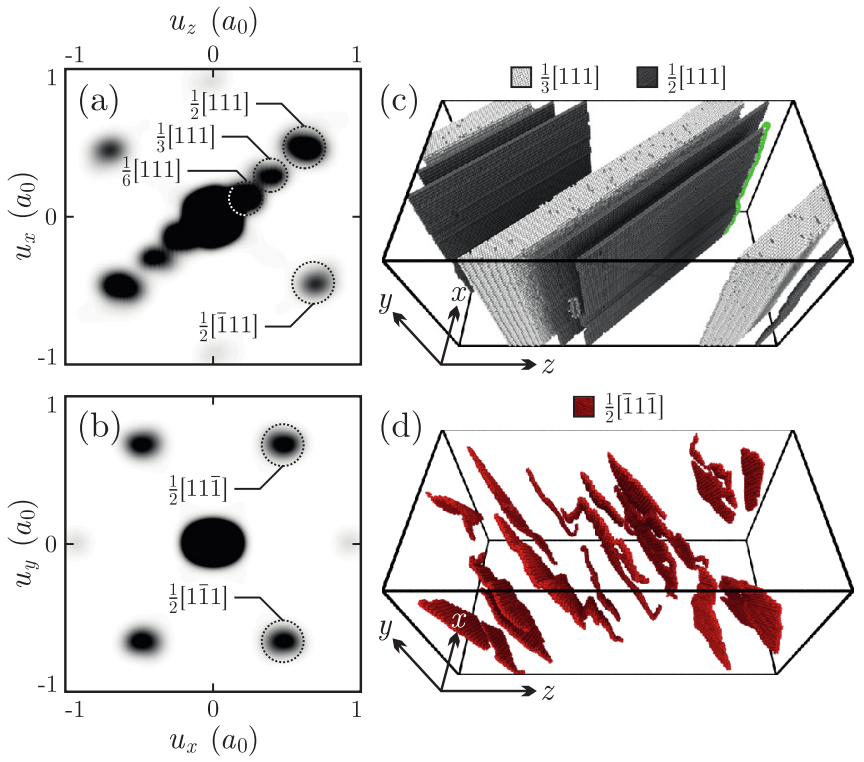

FIG. 4. Visualization of the plasticity mechanisms active in $24.8 \times 24.8 \times 1122 \mathrm{~nm}^{3}$ monocrystalline Ta shocked along [011] to $40 \mathrm{GPa}$ via SVA. (a) Cross-section of the neighbor-displacement density plot in the $x z$ plane, showing plasticity mechanisms belonging to the first stage of deformation. Visible are peaks from dislocation slip (Burgers vectors 1/2 [111] and 1/2 [111]) and deformation twinning (Burgers vector 1/6[111]). Note that the twins generate peaks at both $1 / 6$ [111] and 1/3 [111]; we only use the latter in the SVA algorithm. (c) Atoms contributing to selected peaks in the density plot, which form slip planes (dark grey) and deformation twins (light grey). The dislocation preceding one of the slip planes, detected using the DXA $[62,64]$, can be seen in green at the right of the image. [(b) and (d)] Similar analysis for the second stage of deformation, which is characterized by slip with associated Burgers vectors $1 / 2[11 \overline{1}]$ and $1 / 2[\overline{1} 1 \overline{1}]$.

The first is characterized by a combination of dislocation slip and deformation twinning of the types [111](211) and [111](211). That twinning of this kind operates in rapidly strained [011] Ta is well-known, having been extensively observed in MD [22,27,49,58,65,66] and in experiment, both ex post facto [66-68] and, more recently, in situ via timeresolved XRD [22,27]. The deformation twins nucleate first, and are then partially replaced by the full dislocations, which are able to accommodate the same shear strain as the twins without the large energy cost associated with their surfaces. In our coordinate system, the shearing motion associated with these mechanisms takes place exclusively in the $x z$ plane. The second stage of deformation, whose onset is delayed with respect to the first, involves dislocation slip in directions [111] and $[\overline{1} 1 \overline{1}]$, which are situated in the $x y$ plane (i.e., normal to the shock). Figure 4(d) shows that the slipped atoms left in the wake of these dislocations form a relatively complicated structure. This is because the dislocations mediating slip during this latter stage of flow have predominantly screwlike character, so they are permitted to cross-slip between planes; dislocations with Burgers vector 1/2 [111] , for instance, can

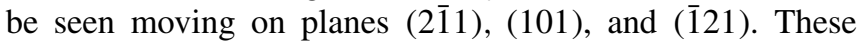
two stages of deformation exhaust the four distinct $\langle 111\rangle$ directions in which the bcc crystal is inclined to flow.

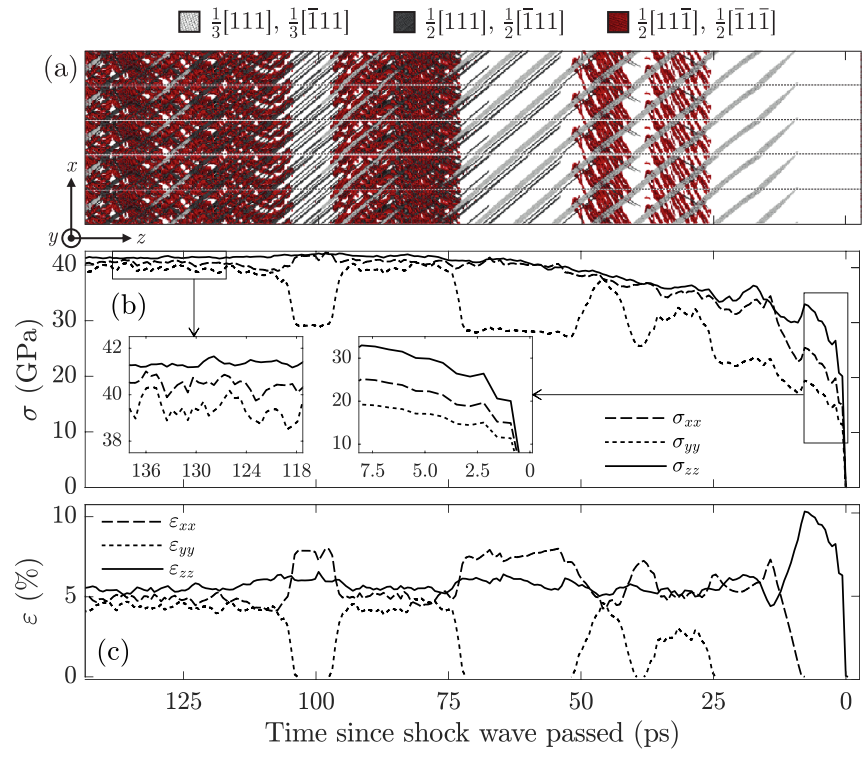

\begin{tabular}{|c|c|c|c|c|c|}
\hline 400 & 500 & 600 & 700 & 800 & 900 \\
\hline
\end{tabular}

FIG. 5. Visualization of the plasticity mechanisms active in monocrystalline Ta shocked to $40 \mathrm{GPa}$ along [011] (the positive $z$ direction), alongside profiles of the stresses and elastic strains. (a)-(c) are plotted on the same scale. The $z$ coordinate is primarily expressed in terms of the local time since the compression front passed; the secondary axis shows the distance from the piston generating the shock. (a) Atoms that have participated in plastic flow events, identified via SVA (as in Fig. 4). Visible are planar deformation twins and slip planes with associated Burgers vectors in the $x z$ plane (light and dark grey, respectively), and nonplanar slip planes with Burgers vectors in the $x y$ plane (red). Periodic images of the cell have been added in the $x$ direction for clarity. (b) Stresses along the computational cell axes averaged over the cross-section of the crystal. Left and right insets show the late-time stresses and stresses within the elastic precursor, respectively. (c) Elastic strains in the computational cell basis. Positive strains denote compression.

The key observation supplied by the SVA is that the crystallographic inequivalence of the crystal's two transverse directions is clearly manifested in the way it plastically deforms: while the first stage of deformation is characterized by flow in the $x z$ plane, there exists no similar mechanism allowing flow in the $y z$ plane-the crystal must instead settle for relatively sedate flow in the $x y$ plane. The transverse components of stress $\sigma_{x x}$ and $\sigma_{y y}$, coupled as they are to separate plasticity mechanisms, must therefore evolve at different rates. To verify this, we show the atoms that have participated in plastic flow events alongside the local components of stress and elastic strain (expressed in the computational cell basis) in Fig. 5. This allows us to give a detailed account of how each plasticity mechanism alters the stresses perpendicular to the shock, and thus how the plastic and elastic aspects of the crystal's deformation are coupled.

The compression wave is led by an elastic precursor within which the crystal suffers uniaxial strain by $10 \%$ along $z$. The normal stresses increase to around half of their final values within this elastic zone in the order $\sigma_{z z}>\sigma_{x x}>\sigma_{y y}$, as is expected from the crystal's elastic 
moduli $[69,70]$. Resolved shear stress (RSS) $\tau$ accumulates most rapidly on the $[111](\overline{2} 11)$ and $[\overline{1} 11](211)$ systems (for which $\tau=\sqrt{2} / 3\left(\sigma_{z z}-\sigma_{x x}\right)$ ), hence the triggering of the first stage of plastic relaxation. The attendant flow in the $x z$ plane allows the crystal to expand along $z$ and collapse along $x$, causing stresses $\sigma_{x x}$ and $\sigma_{z z}$ to converge; the lattice spacing along $y$, meanwhile, remains unchanged. The systems under the greatest RSS following the first stage of deformation are $[11 \overline{1}](\overline{2} 1 \overline{1})$ and $[\overline{1} 1 \overline{1}](21 \overline{1})$ [for which $\tau=\sqrt{2} / 3\left(\sigma_{x x}-\sigma_{y y}\right)$ ], followed closely by the set [11 $\overline{1}](1 \overline{1} 0),[11 \overline{1}](\overline{1} 0 \overline{1})$, [ $\overline{1} 1 \overline{1}](\overline{1} \overline{1} 0)$, and [ $\overline{1} 1 \overline{1}](\overline{1} 01)(\tau=$ $\left.1 / \sqrt{6}\left(\sigma_{x x}-\sigma_{y y}\right)\right)$, i.e., those involving transverse slip; these shear stresses are actually enhanced beyond the values they assume within the precursor by the first deformation mode, which causes $\sigma_{x x}$ to increase. The transverse slip systems become active several tens of picoseconds after the first set and allow the crystal to collapse along the $y$ direction, thus relieving most of the remaining shear stress that the first stage of deformation could not. The stress state to which the fully relaxed portion of the crystal converges is slightly nonhydrostatic, supporting residual shear stresses of order $1 \mathrm{GPa}$ [see the left inset of Fig. 5(b)]. The difference between the transverse stresses $\sigma_{x x}-\sigma_{y y}$ is thus finite throughout the deformation, and can in fact reach values as extreme as $10 \mathrm{GPa}$ in-between the two stages of deformation.

At higher shock pressures, the response of the crystal is qualitatively similar: flow takes place rapidly in the [111] and

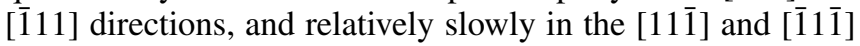
directions. There do exist differences in certain aspects of the deformation as the shock pressure increases; for instance, the delay between the onset of the first and second stages of deformation shrinks to zero. We also note that the number of stable deformation twins rapidly decreases, as observed by Ravelo et al. [49], and dislocation slip comes to be the dominant means of plastic relaxation. However, the crystal's underlying twofold rotational symmetry about $z$ is obviously preserved, and so it continues to assume an anisotropic transverse stress state during and after plastic relaxation up to pressures as high as 1 Mbar. In Fig. 6, we show the average deviatoric stresses and engineering elastic strains, defined by $\sigma^{\prime}=\sigma-$ $1 / 3 \operatorname{Tr}[\sigma] I$ and $\varepsilon^{\prime}=\varepsilon-1 / 3 \operatorname{Tr}[\varepsilon] I$, respectively, within the fully relaxed region of monocrystals compressed to between 40 and $100 \mathrm{GPa}$. The steady-state transverse stress anisotropy $\sigma_{x x}-\sigma_{y y}$ over this pressure range takes values between 1.0 and $1.5 \mathrm{GPa}$. This anisotropy manifests in the elastic strain state of the crystal as a persistent difference between $\varepsilon_{x x}$ and $\varepsilon_{y y}$ whose magnitude (approximately $0.5 \%$ ) is comparable to the traditional shear strain $\varepsilon_{S}-\varepsilon_{T} \equiv \varepsilon_{z z}-\left(\varepsilon_{x x}+\varepsilon_{y y}\right) / 2$. Further reduction of the stress anisotropy is prevented by material strength, or more specifically the shear stress required to effect plastic flow along [11ī] and [i111] .

To summarize, single-crystal tantalum shocked along [011] generally assumes an anisotropic stress state in the plane normal to the shock, due to each component of transverse stress being coupled to different plasticity mechanisms. The anisotropy may reach values as high as $10 \mathrm{GPa}$ during the course of plastic relaxation, and falls to no lower than $1 \mathrm{GPa}$ in the steady-state due to material strength. We now go on to consider the consequences of transverse stress anisotropy at

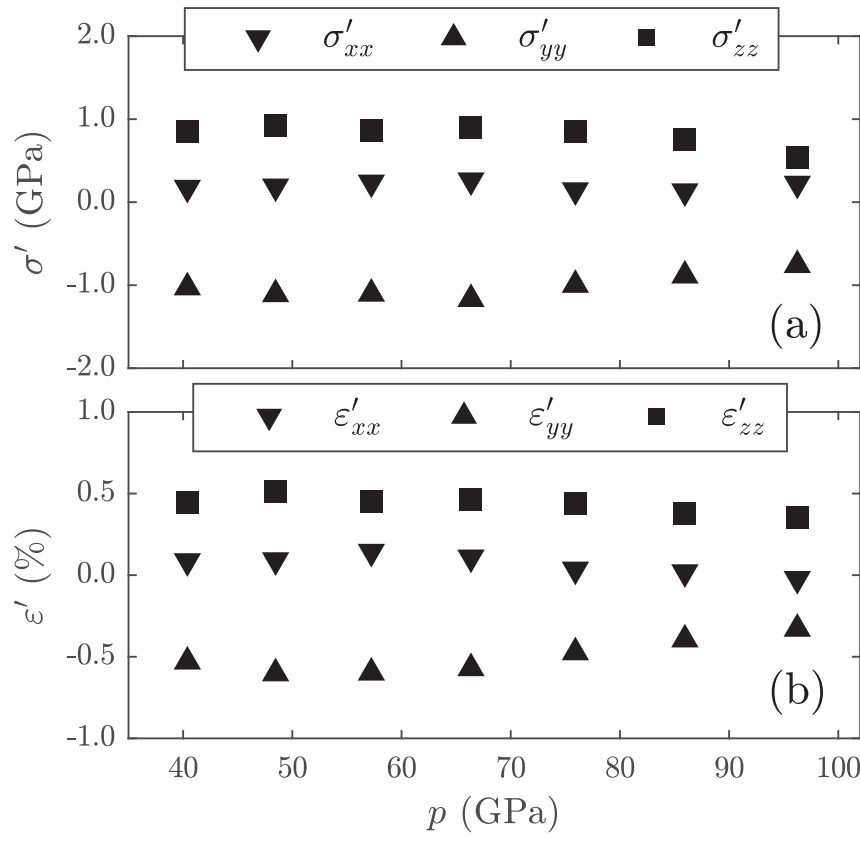

FIG. 6. (a) Steady-state deviatoric stresses of monocrystalline Ta shocked along [011] to between 40 and $100 \mathrm{GPa}$. (b) Corresponding deviatoric elastic strains.

the level of a single grain for the evolution of a polycrystal composed of many such grains.

\section{B. Polycrystal response below the HEL}

We showed in Sec. III A that a laterally confined Ta crystal shocked above its HEL along [011] will reach a state in which $\sigma_{x x} \neq \sigma_{y y}$. Suppose we were to construct a columnar polycrystal from several such compressed monocrystals by rotating each through some angle about $z$ and then tessellating them in the $x y$ plane. It is clear that such a polycrystal would in general be mechanically unstable: if one were to move along any given direction in the $x y$ plane, the normal stress components $\sigma_{x x}$ and $\sigma_{y y}$ would change whenever one passed from one grain to another, because each grain is anisotropic in the transverse plane. In other words, the presence of transverse stress anisotropy at the level of each individual grain necessarily implies the existence of stress gradients over the grain boundaries. The polycrystal would thus fail to satisfy the stability criterion $\nabla \cdot \sigma=0$ in the vicinity of the grain boundaries, causing nearby material to experience a net force and therefore accelerate. The equilibrium stress state of grains in the polycrystal must therefore differ from the state they assume in isolation. Moreover, one should expect the stress gradients over the grain boundaries in such a polycrystal to temporarily assume extremely large values during the course of its deformation, causing neighboring grains to interact strongly with one another. To elucidate this basic physics, we first study the response of a polycrystal loaded below its HEL.

We uniaxially compressed a $46.7 \mathrm{~nm}$ long, fully periodic supercell with the CB geometry by 5\% along the $z$ direction by instantly rescaling every atom's $z$ coordinate by 0.95 . The cell was simulated under an NVE ensemble for $200 \mathrm{ps,}$ during which time it oscillates about a mechanically stable 


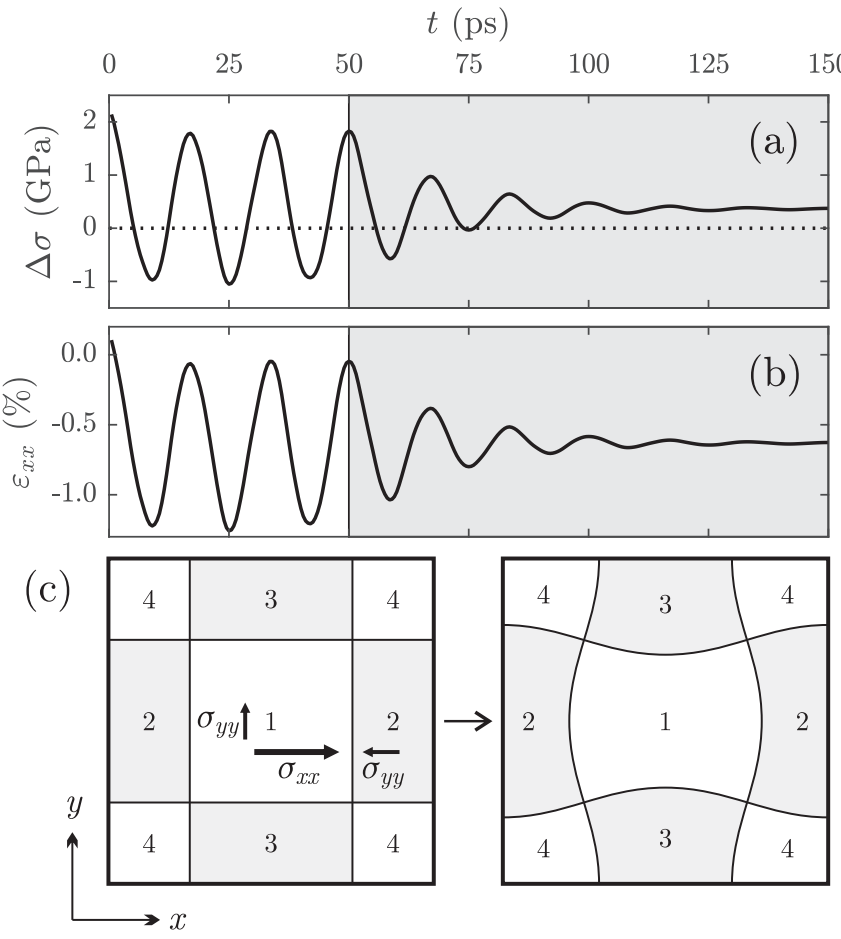

FIG. 7. (a) Transverse stress anisotropy $\Delta \sigma=\sigma_{x x}-\sigma_{y y}$ for grain 1 of a $\mathrm{CB}$ polycrystal instantaneously strained by $5 \%$ along $z$ at $t=0$ ps. (b) Transverse elastic strain component $\varepsilon_{x x}$ for the same grain. Note that $\varepsilon<0$ denotes expansion. The shaded regions indicate where oscillation of the grains is damped by a Langevin thermostat. (c) Schematic cross-section of the CB polycrystal at $t=0$ and $150 \mathrm{ps}$ (left and right images, respectively), showing the (greatly exaggerated) expansion of each grain along its local [100] axis in response to the stress gradients induced over their boundaries by the compression.

state (as we explain below). To hasten the convergence of the crystal to its steady state, a weak Langevin thermostat was applied after several tens of picoseconds, which damps out large-scale oscillations while maintaining the system's temperature.

Shown in Figs. 7(a) and 7(b) are the difference between the spatially averaged transverse stresses, $\Delta \sigma=\sigma_{x x}-\sigma_{y y}$, and the transverse elastic strain component $\varepsilon_{x x}$ for the central grain of the supercell as functions of time. We see that the transverse stress anisotropy $\Delta \sigma$ oscillates about a value close to zero, which can be understood as follows. The compression of the crystal at $t=0$ instantaneously effects a stress state in which $\Delta \sigma=2.1 \mathrm{GPa}$. That the two transverse stresses are unequal is a consequence of the transverse directions ([100] and [01 $\overline{1}]$ ) being crystallographically inequivalent; identical behavior was seen in the elastic precursor of shock-compressed single crystals in Sec. III A. Now, the rotational symmetry of the $\mathrm{CB}$ geometry is such that $\sigma_{y y}$ in grain 1 is equal to $\sigma_{x x}$ in grain 2, as indicated schematically in Fig. 7(c). There thus exist at $t=0$ ps stress gradients over the grain boundaries. Atoms on the boundaries between grains 1 and 2 therefore experience a net force in the $x$-direction. Consequently grain 1 expands along its [100] axis, allowing $\sigma_{x x}$ to decrease. By symmetry, the central grain also contracts along the $y$ direction (since grain 3 expands along its own [100] axis), causing $\sigma_{y y}$ to increase. The effect of this transverse elastic deformation is to relax the stress gradients over the grain boundaries, and in so doing cause $\sigma_{x x}$ and $\sigma_{y y}$ to converge. The grain then overshoots the state of mechanical equilibrium (for which $\Delta \sigma \approx 0 \mathrm{GPa}$ ) due to its inertia, and subsequently executes something close to simple harmonic motion (SHM). Once the motion has been damped out by the thermostat, the residual stress state in each grain is such that $\Delta \sigma$ is only $0.38 \mathrm{GPa}$.

What we are seeing here is the relief of shear stress not by plastic, but elastic deformation. This means of relaxation is available only to a polycrystal, for the following reasons. Both a single crystal and a polycrystal that are laterally confined have fixed transverse dimensions. For the single crystal, this means that any change in transverse lattice spacing is necessarily reliant on plastic flow; only by changing the number of crystal planes can their spacing be made to change. The polycrystal, by contrast, is subject to no such limitation: individual grains may undergo elastic strain transverse to the shock without the aid of plasticity mechanisms, provided they do so in such a way that the sum of their strains vanishes. In this instance, grain 1 is allowed to expand along [100] by grain 2's contraction along [01 1 ]. Note also that this elastic deformation is able to relax the transverse stress anisotropy to a greater degree than the corresponding plasticity mechanism can: while $\Delta \sigma$ was limited to at least $1.0 \mathrm{GPa}$ for the single crystals studied in Sec. III A (specifically by the

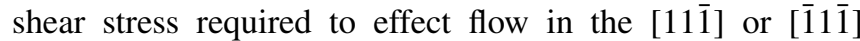
directions), the difference here is no more than $0.4 \mathrm{GPa}$. The polycrystal can thus reach a state of lower shear stress than the individual grains of which it is composed could in isolation.

However, while it is more efficient than plastic flow, the elastic relaxation mechanism cannot eliminate the transverse stress anisotropy entirely. To understand why this is, we need to consider the full stress state of the crystal. In Fig. 8, we show the variation in the $x y$ plane of not only $\varepsilon_{x x}$ and $\sigma_{x x}$ but also the off-diagonal stress tensor component $\sigma_{x y}$, before and after the stress gradients have relaxed. We see that as the supercell relaxes the transverse strain field $\varepsilon_{x x}$ becomes less homogeneous, the transverse stress $\sigma_{x x}$ more so-the system moves from the isostrain or Voigt condition [71] towards the isostress or Reuss limit [72]. Yet, while the difference between $\sigma_{x x}$ in adjacent grains clearly decreases following relaxation, it does not vanish. In fact, towards the upper and lower boundaries of each grain, $\sigma_{x x}$ differs only slightly from the value it assumed before relaxation. This frustration is caused by material either side of the grain boundaries being driven in different directions: grain 1, for instance, "wants" to expand along $x$, while grain 3 wants to contract. The result is the generation of shear (off-diagonal) stresses in the vicinity of the boundary that are particularly strong at the corners of the grains, where the spatial gradients of the atomic displacement field are greatest. A thin material element straddling a grain boundary therefore experiences additional tractions on those of its faces that are perpendicular to the boundary; an example of such an element is depicted in Fig. 8 at the boundary between grains 1 and 2 . The tractions provide a countervailing force that opposes the normal stress gradient 

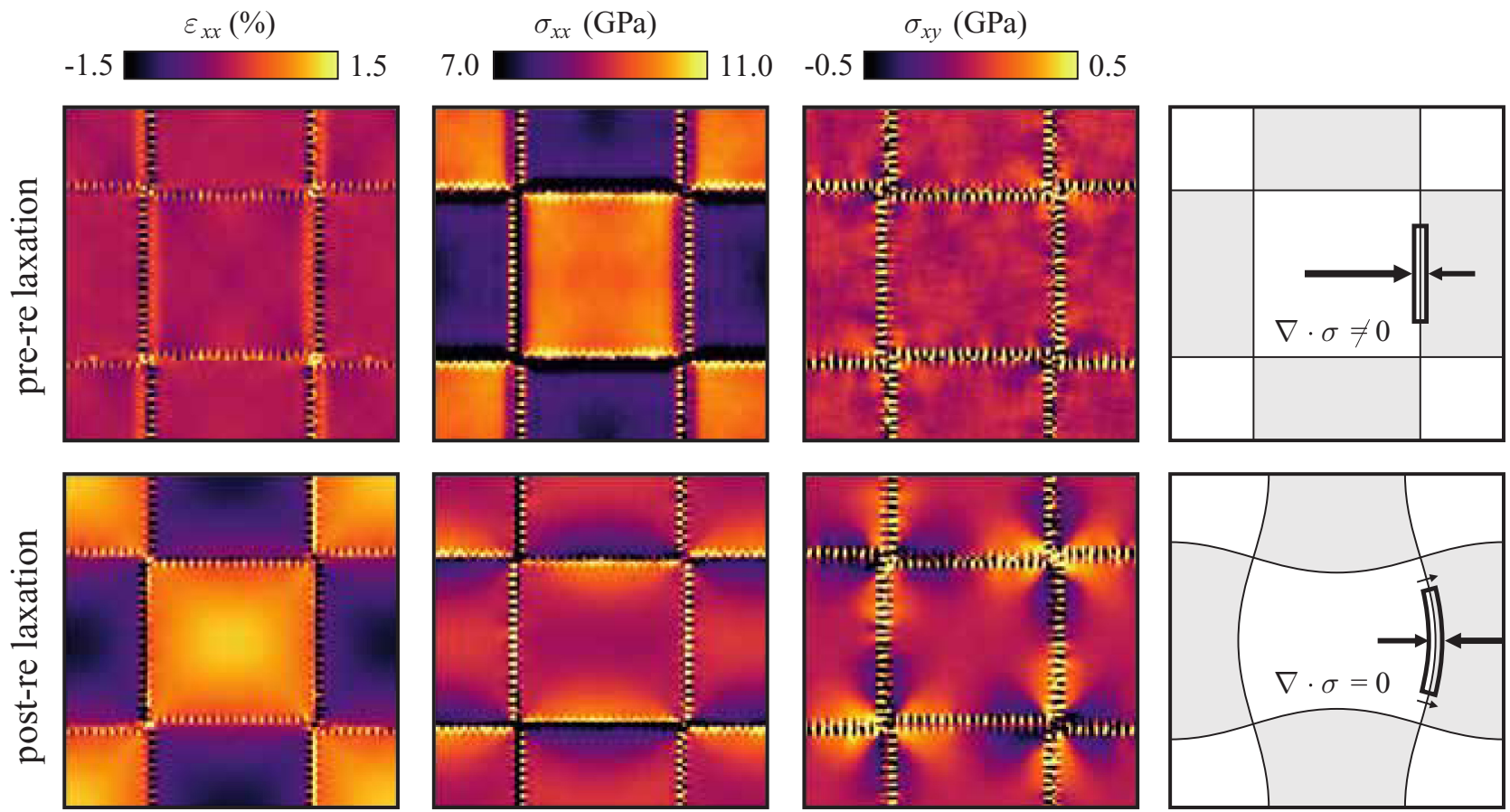

FIG. 8. Cross-sections of a CB polycrystal instantaneously strained by $5 \%$ along $z$ at $t=0$ ps both before and after relaxation of the resultant stress gradients (upper and lower rows, respectively), showing spatial variation of the fields $\varepsilon_{x x}, \sigma_{x x}$, and $\sigma_{x y}$. Panes in the rightmost column depict the traction acting on a material element straddling the grain boundary between grains 1 and 2 before and after the grains have undergone their (greatly exaggerated) deformation.

over the boundary, such that

$$
[\nabla \cdot \sigma]_{x}=\partial_{x} \sigma_{x x}+\partial_{y} \sigma_{x y}=0 .
$$

The crystal is thus able to tolerate normal stress gradients over its grain boundaries, which frustrates relaxation of the transverse stress anisotropy $\Delta \sigma$.

We further note that the efficiency of the elastic relaxation mode (i.e., how far short of the Reuss limit the supercell falls) depends on the geometry of the polycrystal. Shown in Fig. 9 is a comparison of the off-diagonal stress field $\sigma_{x y}$ for the CB and $\mathrm{HC}$ geometries post-relaxation. Note that $\sigma_{x y}$ in the central grain is both less localized and of greater magnitude in the $\mathrm{HC}$ geometry than in the $\mathrm{CB}$. The typical normal stress gradients $\partial_{x} \sigma_{x x}$ and $\partial_{y} \sigma_{y y}$ required to balance gradients in $\sigma_{x y}$ are therefore correspondingly larger, meaning relaxation of $\Delta \sigma$ is frustrated to a greater degree-the average transverse stress anisotropy in the $\mathrm{HC}$ geometry falls to only $0.66 \mathrm{GPa}$. The increased frustration suffered by grains in the $\mathrm{HC}$ geometry is caused by their deformation being constrained by the presence

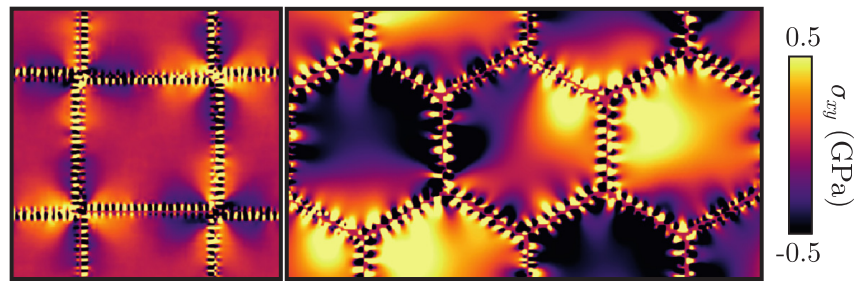

FIG. 9. Comparison of the off-diagonal stress field $\sigma_{x y}$ for CB (left) and $\mathrm{HC}$ (right) polycrystals strained by 5\% along $z$ following relaxation of the induced stress gradients. of six (rather than only four) neighbors. The elastic relaxation mechanism in the $\mathrm{HC}$ is, however, still more efficient than the corresponding plasticity mechanism.

Finally, it is important to appreciate the timescale $P$ over which the elastic deformation occurs. Figure 7 showed that the polycrystal adapts to the imposed stress gradients within 5 ps. To identify the property of the system that allows it to respond this rapidly, we can use simple dimensional arguments to construct the functional form of $P$. The relaxation timescale must depend on the elastic constants $\left\{c_{i j}\right\}$, as these determine the magnitude of the stresses induced in the system when it is deformed to a given extent; it must also depend on the mass density $\rho$, as this dictates the rate at which the system can locally respond to stresses of a given magnitude; and it must depend on the transverse dimension of the grains, $L$, because it takes a finite time for the stress waves generated at the grain boundaries to propagate into the bulk of the grains. The only dimensionally consistent combination of these variables reads

$$
P=\sqrt{\frac{\rho}{\sum_{i j} \alpha_{i j} c_{i j}}} L,
$$

where $\left\{\alpha_{i j}\right\}$ is a set of dimensionless constants, presumably of order unity. Of the variables appearing in Eq. (11), $L$ is arguably the most important. For most metals, the square root (which strongly resembles the inverse of a sound speed), ranges between $10^{-3} \mathrm{sm}^{-1}$ (e.g., for lead [73]) and $10^{-4}$ $\mathrm{sm}^{-1}$ (e.g., for beryllium [74]), and cannot be pushed far beyond this range by shock compression unless the loading is extreme (i.e., to several terapascals [75]). The grain size, meanwhile, can in principle span three orders of magnitude, from nanometers to micrometers, depending on the way the 
(a)
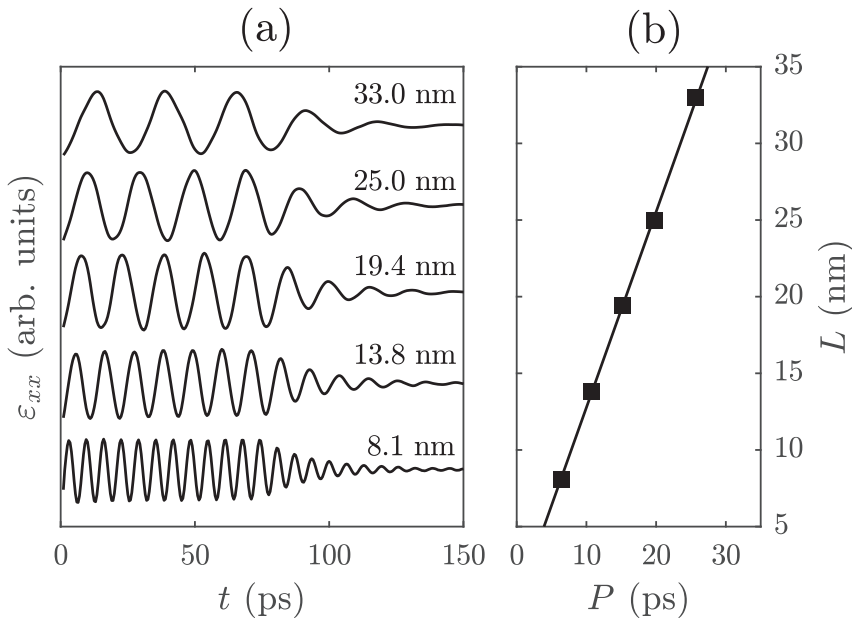

FIG. 10. (a) Evolution of the transverse strain component $\varepsilon_{x x}$ for the central grain of uniaxially strained $\mathrm{CB}$ polycrystals of various sizes. Each profile, which is labeled by the corresponding grain size $L$, has been vertically offset for clarity. Motion is damped by a Langevin thermostat after 75 ps. (b) Oscillation period $P$ as a function of grain size $L$. Shown also is the line of best fit obtained from a linear least-squares regression analysis with intercept fixed at zero.

polycrystal is prepared. It is therefore $L$ that is primarily responsible for setting the relaxation timescale, which for a nanocrystalline system is of order picoseconds.

To verify the linear dependence of $P$ on $L$ suggested by dimensional analysis, we performed a set of instantaneous compression simulations similar to those discussed above on CB supercells with grain sizes in the range $8.1 \mathrm{~nm} \leqslant L \leqslant$ $33.0 \mathrm{~nm}$. The variation of $\varepsilon_{x x}$ with time for grain 1 is shown for each of these supercells in Fig. 10(a). By Fourier transforming each strain profile and identifying the frequency component with the greatest intensity, we computed the variation of the oscillation period $P$ with $L$. The results, plotted in Fig. 10(b), show that the proportionality predicted by Eq. (11) is indeed borne out in the simulations.

That the grains in an elastically compressed nanocrystal interact over only a few picoseconds is significant, because plastic flow takes place in a shock-loaded single crystal over a comparable timescale. If the nanocrystal were loaded above its HEL, then, these two mechanisms would take place concurrently. As we will show in Sec. IIIC, this allows the mechanisms to combine "nonlinearly," in the sense that the elastic deformations can actually activate or deactivate plasticity mechanisms, depending on the shock pressure.

In summary, we found that when a columnar polycrystal is uniaxially compressed below its HEL, its constituent grains elastically expand and contract normal to the loading axis in order to relax the stress gradients that are induced over its grain boundaries. Relaxation of these stress gradients reduces the degree of transverse stress anisotropy $\Delta \sigma=\sigma_{x x}-\sigma_{y y}$ within each grain. The efficiency of this elastic relaxation is limited to a greater extent in the $\mathrm{HC}$ than the CB geometry, but for both geometries this mechanism still relieves transverse shear stress more efficiently than the corresponding plastic deformation mode can. The nanocrystals studied here relax within picoseconds, i.e., at a rate comparable to that of plastic flow. We will now consider the full elastoplastic response of columnar polycrystals shock-compressed above their HEL.

\section{Polycrystal response above the HEL}

Having seen how a single grain undergoes plastic deformation in isolation, and how a polycrystal responds to transverse stress anisotropy, we are in a position to discuss the full elastoplastic response of a shock-compressed polycrystal. We will describe the most conspicuous features of the crystal's dynamic response, first just above the single-crystal HEL at $40 \mathrm{GPa}$, and then at higher pressures of $60 \mathrm{GPa}$ and above. We will then compare the equilibrium mechanical state of the $\mathrm{CB}$ and $\mathrm{HC}$ polycrystals with that of the isolated single crystals.

\section{Slip deactivation at low pressure}

It was shown in Sec. III B that grains in an elastically loaded, fiber-textured, columnar polycrystal deform normal to the loading axis due to the stresses they exert on one another. These interactions lead directly to relief of the shear stress associated with the transverse stress anisotropy $\Delta \sigma$. This same shear stress was relieved by dislocation slip in the [111] and $[\overline{1} 1 \overline{1}]$ directions in the isolated grains discussed in Sec. III A. Dislocation slip of this kind and intergranular interactions therefore act in competition when the polycrystal is shock-compressed above its HEL. To demonstrate this, we show in Fig. 11 the slipped atoms within a cross-section of a CB polycrystal shock-compressed to $40 \mathrm{GPa}$, alongside plots of the stress and elastic strain variation along the length of the central grain. The wave structure is substantially different to that within the single crystal shocked to the same pressure, as we now go on to explain.

Within the elastic precursor, the normal stresses initially increase in the order $\sigma_{z z}>\sigma_{x x}>\sigma_{y y}$, exactly as they did in the single crystal. However, the transverse stresses $\sigma_{x x}$ and $\sigma_{y y}$ almost immediately start to converge due to the grains interacting with one another; in response to the stress gradients over its boundaries, grain 1 expands along $x$, and contracts along $y$. At the rear of the elastic zone, the average transverse stress anisotropy $\Delta \sigma$ has vanished entirely due to this elastic relaxation, as shown in the right inset of Fig. 11(b). The crystal subsequently undergoes slip and twinning in the [111] and [111] directions due to the difference between $\sigma_{x x}$ and $\sigma_{z z}$, just as the single crystal did, causing the crystal to collapse along $x$ (i.e., the [100] direction). This deformation mechanism alone is sufficient to relieve the majority of the remaining shear stress, and so no further plastic flow takes place. The second stage of deformation that was observed in the isolated single crystals is thus conspicuously absent.

The fact that the CB polycrystal does not yield in the [111] and $[\overline{1} 1 \overline{1}]$ directions at low shock pressure is a consequence of intergranular interactions. Since the interactions cause $\sigma_{x x}$ and $\sigma_{y y}$ to converge, and thus serve the same purpose as slip along [11 $\overline{1}]$ and $[\overline{1} 1 \overline{1}]$, they render these latter plasticity mechanisms unnecessary. Phrased another way, the collapse along the $y$ direction (i.e., [011] ) that was permitted in the isolated grains by the second stage of plastic deformation can instead be realized by purely elastic compression of each grain by its neighbors. Note that the elastic relaxation mechanism is only able to replace plastic flow because it takes place so 

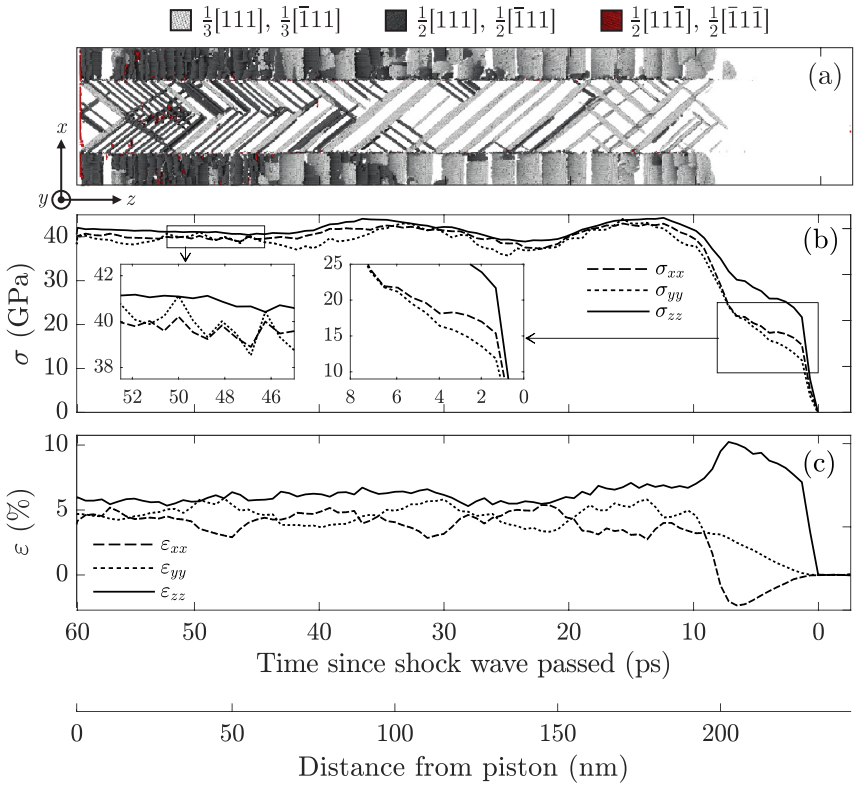

FIG. 11. Visualization of the plasticity mechanisms active in polycrystalline $\mathrm{Ta}$ with the $\mathrm{CB}$ geometry shocked to $40 \mathrm{GPa}$ along [011] (the positive $z$ direction), alongside profiles of the stresses and elastic strains. (a)-(c) are plotted on the same scale. The $z$ coordinate is primarily expressed in terms of the local time since the compression front passed; the secondary axis shows the distance from the piston generating the shock. (a) Atoms that have participated in plastic flow events, identified via SVA (as in Fig. 4). Visible are planar deformation twins and slip planes with associated Burgers vectors in the $x z$ plane (light and dark grey, respectively). The central grain is grain 1, that on the outside is grain 2. (b) Stresses along the computational cell axes averaged over the cross-section of the central grain. Left and right insets show the late-time stresses and stresses within the elastic precursor, respectively. (c) Elastic strains in the central grain.

quickly - the nucleation of $1 / 2$ [1111] and $1 / 2$ [ $\overline{1} 1 \overline{1}]$ dislocations takes at least $25 \mathrm{ps}$ at $40 \mathrm{GPa}$, while the grains can elastically deform normal to the shock within 5 ps.

The second stage of plastic deformation is also suppressed in the HC geometry, though to a lesser extent. While 1/2 [11 1] and $1 / 2[\overline{1} 1 \overline{1}]$ dislocations are almost entirely absent in the CB geometry, there do exist several such dislocation loops stemming from the grain boundaries in the HC geometry. However, their growth is quickly arrested before they can traverse the grains by the shear stress relief provided by elastic deformation, so the bulk of the grains remain devoid of $1 / 2$ [11 1$]$ and $1 / 2[\overline{1} 1 \overline{1}]$ dislocations, as we show in Fig. 12. The fact that grains in the HC geometry have to rely to some extent on "conventional" plastic deformation is consistent with the observation made in the Sec. III B that elastic deformation is less efficient at relieving shear stress in this latter geometry.

In brief, we find that a polycrystal in the $\mathrm{CB}$ geometry shock-loaded just above the single-crystal HEL does not suffer dislocation slip in the [ $\overline{1} 11]$ and $[\overline{1} 1 \overline{1}]$ directions. These plasticity mechanisms are replaced by the elastic deformation effected by intergranular interactions, which relieves the same shear stress over a shorter timescale. Both mechanisms are active to some degree in the $\mathrm{HC}$ geometry due to the reduced efficiency of elastic deformation, but the total amount of
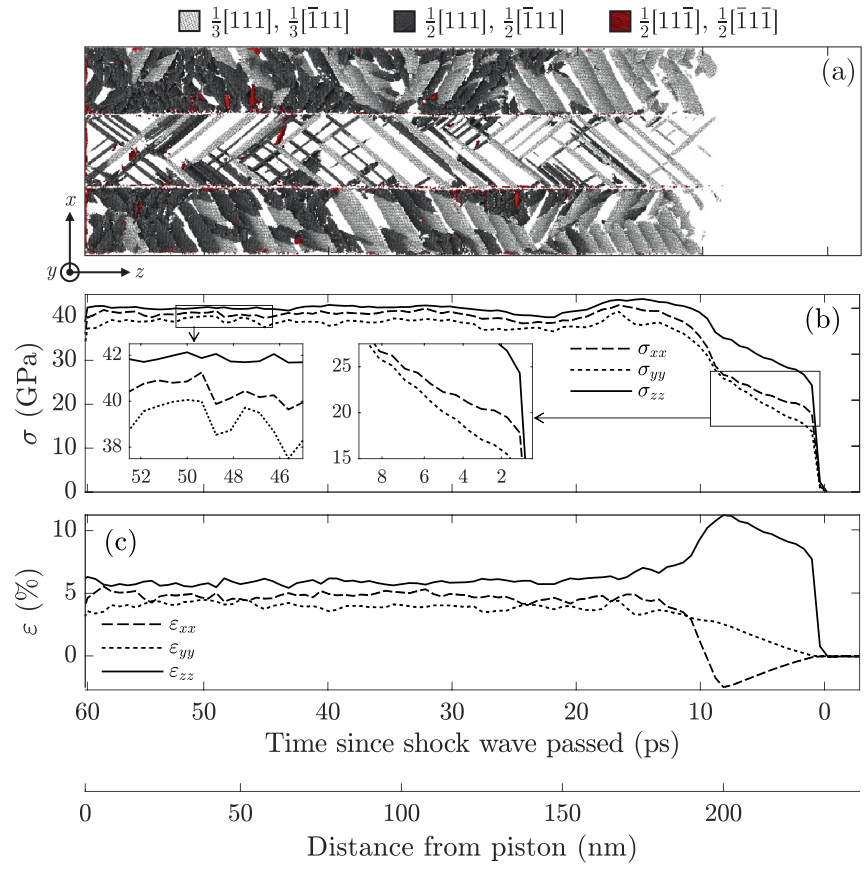

FIG. 12. Visualization of the plasticity mechanisms active in polycrystalline Ta with the HC geometry shocked to $40 \mathrm{GPa}$ along [011] (the positive $z$ direction), alongside profiles of the stresses and elastic strains. (a)-(c) are plotted on the same scale. The $z$ coordinate is primarily expressed in terms of the local time since the compression front passed; the secondary axis shows the distance from the piston generating the shock. (a) Atoms that have participated in plastic flow events, identified via SVA (as in Fig. 4). Visible are planar deformation twins and slip planes with associated Burgers vectors in the $x z$ plane (light and dark grey, respectively), and, to a limited degree, nonplanar slip planes with associated Burgers vectors in the $x y$ plane (red). (b) Stresses along the computational cell axes averaged over the cross-section of the central grain. Left and right insets show the late-time stresses and stresses within the elastic precursor, respectively. (c) Elastic strains in the central grain.

plastic flow is still substantially lower than that in the isolated single crystals.

\section{Twinning activation at higher pressure}

It was noted in Sec. III B that the stress gradients formed over the grain boundaries in an elastically compressed polycrystal do not decrease monotonically in magnitude, but oscillate about their equilibrium values due to the inertia of the grains. It is thus possible for the transverse stress anisotropy $\Delta \sigma$, and so the shear stress associated with it, to temporarily change sign during the course of the deformation. If the crystal is shocked with sufficient strength, the magnitude of this inverted shear stress can become great enough to trigger a new variant of deformation twinning.

Shown in Fig. 13(a) is a cross-section of the neighbordisplacement density plot in the $x y$ plane taken from a $\mathrm{CB}$ polycrystal shock-compressed to $60 \mathrm{GPa}$. The clearest features of the plot are the eight peaks surrounding the origin that correspond to transverse slip in the [11 $\overline{1}]$ and $[\overline{1} 1 \overline{1}]$ directions. The four peaks for which $\left|u_{y}\right|>\left|u_{x}\right|$ (which were previously observed in Fig. 4 for the single crystal) come from grains 

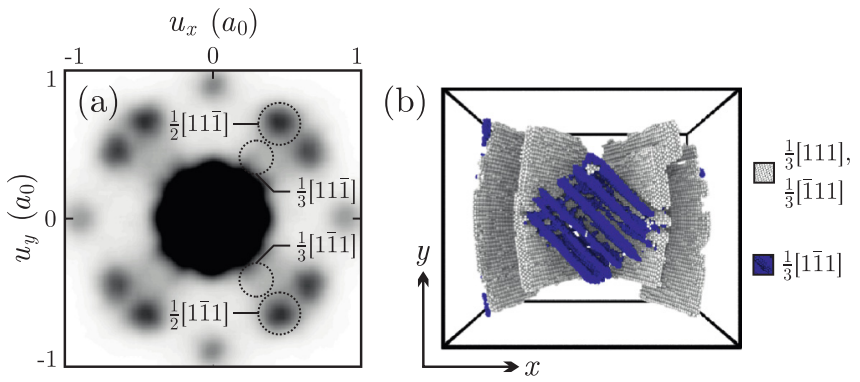

FIG. 13. Visualization of deformation twins in polycrystalline Ta with the CB geometry shocked along [011] to $60 \mathrm{GPa}$. (a) Crosssection of the neighbor-displacement density plot in the $x y$ plane.

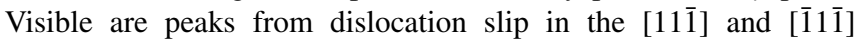
directions and (very weakly) the new, transverse deformation twins with Burgers vectors in those same directions. Note the plot is a superposition of slip vectors from all four grains, hence its approximate fourfold rotational symmetry about the origin. (b) Atoms forming the transverse twins (blue) alongside the conventional twins with habit planes ( 211$)$ and (211) (white) as viewed along the loading axis, identified not via SVA but the template-matching technique described in Sec. II C.

1 and 4, the other four from grains 2 and 3. In addition to these peaks, it is also possible (though barely) to discern patches of intensity at locations corresponding to $1 / 3$ [111] and $1 / 3[\overline{1} 1 \overline{1}]$, suggesting that deformation twinning of a previously unobserved type is taking place. However, the contrast of these twin peaks at this high shock pressure is extremely poor (for the reasons discussed in Sec. II B), making reliable identification of the twinned atoms via the SVA technique impractical. For this reason, we identify the new "transverse twins" with the complementary template-matching technique described in Sec. II C, assuming habit planes $(\overline{2} 1 \overline{1})$ and $(21 \overline{1})$. The atoms constituting the transverse twins in the central grain are shown in blue in Fig. 13(b), alongside the conventional twins with habit planes $(\overline{2} 11)$ and (211) for reference. Note that the transverse twins form only near the center of the grain.

To understand how these twins nucleate, we need to understand how the shear stress applied to their habit planes varies in the wake of the shock. In Fig. 14, we show once again the slipped and twinned atoms within a cross-section of the $\mathrm{CB}$ polycrystal shocked to $60 \mathrm{GPa}$ on the same scale as the stress and elastic strain profiles for host atoms in the central grain. Just behind the compression front, $\sigma_{z z}$ and $\sigma_{x x}$ increase at nearly the same rate, their difference being kept small by continuous plastic flow in the [111] and [111] directions, while $\sigma_{y y}$ "lags" as usual due to there being no flow with a component in the $y$ direction. As before, the central grain elastically expands along $x$ and contracts along $y$ in response to the huge stress gradients formed over the grain boundaries, which assume peak values of $10 \mathrm{GPa}$ at around 5 ps compression time, allowing $\sigma_{y y}$ to converge to $\sigma_{x x}$. The grain continues to expand once $\sigma_{x x}-\sigma_{y y} \approx 0 \mathrm{GPa}$ due its inertia, and so the transverse stress anisotropy changes sign, as shown in the right inset of Fig. 14(b). However, rather than reaching $-10 \mathrm{GPa}$, as might be expected from the SHM-like behavior of the elastically compressed supercells described in Sec. III B, the growth of $\Delta \sigma$ is suddenly arrested at 10 ps once it reaches $-2 \mathrm{GPa}$. At this instant, the resolved shear stress
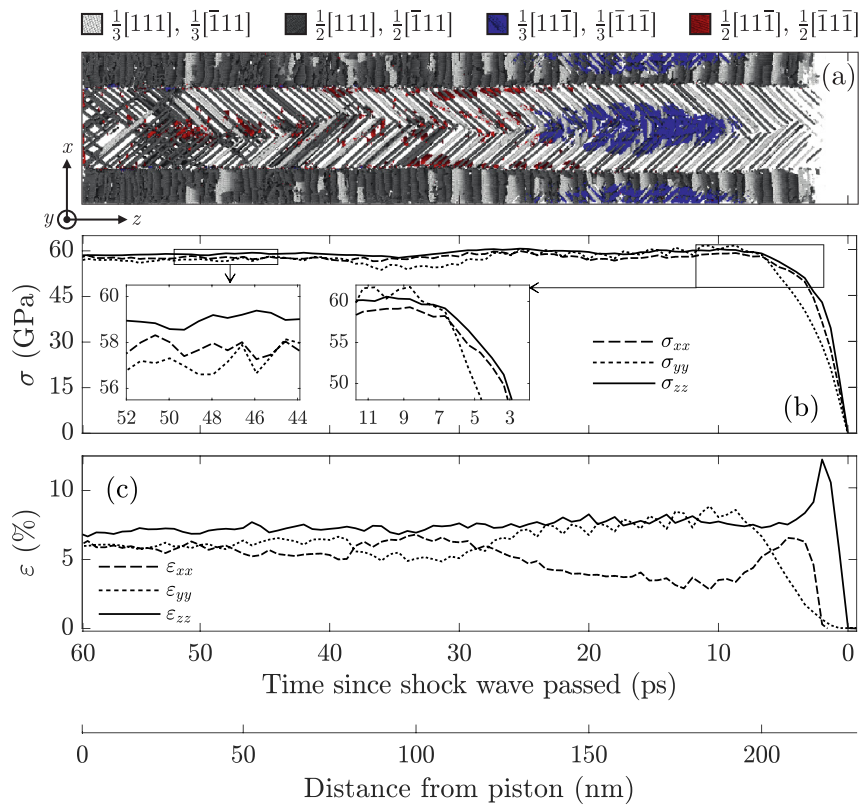

FIG. 14. Visualization of the plasticity mechanisms active in polycrystalline $\mathrm{Ta}$ with the $\mathrm{CB}$ geometry shocked to $60 \mathrm{GPa}$ along [011] (the positive $z$ direction), alongside profiles of the stresses and elastic strains. (a)-(c) are plotted on the same scale. The $z$ coordinate is primarily expressed in terms of the local time since the compression front passed; the secondary axis shows the distance from the piston generating the shock. (a) Atoms that have participated in plastic flow events, identified via SVA (as in Fig. 4). Visible are planar deformation twins and slip planes with associated Burgers vectors in the $x z$ plane (light and dark grey, respectively), and deformation twins with Burgers vectors in the $x y$ plane (blue), present between 130 and $180 \mathrm{~nm}$ from the piston. (b) Stresses along the computational cell axes averaged over the cross-section of the central grain. Left and right insets show the late-time stresses and inversion of the stress state, respectively. (c) Elastic strains in the central grain.

acting on the $(\overline{2} 1 \overline{1})$ and $(21 \overline{1})$ planes is of sufficient magnitude to trigger transverse deformation twinning.

It is the reversal of the sign of $\Delta \sigma$, then, that causes the transverse twins to form. That the crystal refuses to twin when $\Delta \sigma=10 \mathrm{GPa}$, but twins profusely the moment it reaches $-2 \mathrm{GPa}$, is consistent with the so-called twinningantitwinning asymmetry that is characteristic of bcc materials [76-79]. If the crystal were to relieve transverse shear stress via deformation twinning when $\sigma_{x x}>\sigma_{y y}$, it would have to do so via the antitwinning path, in which adjacent atomic planes move through a distance $\|1 / 3[111]\|$. Antitwinning is acted against by a large energy barrier, and is thus suppressed under typical stress conditions in Ta loaded along [011]. If ever $\sigma_{y y}$ exceeds $\sigma_{x x}$, though, the sense of the shear stress is such that flow would instead be realized via the twinning path. Deformation twinning in this sense, which requires motion through only $\|1 / 6[111]\|$, has a comparatively small activation energy [49], and thus ensues copiously in the inverted transverse stress state. The process can be understood perhaps most intuitively with an analogy: conventional deformation twins, with habit planes $(\overline{2} 11)$ and (211), are formed when the grains are compressed along their [011] axis by the shock wave; in much the same way, transverse deformation twins, 

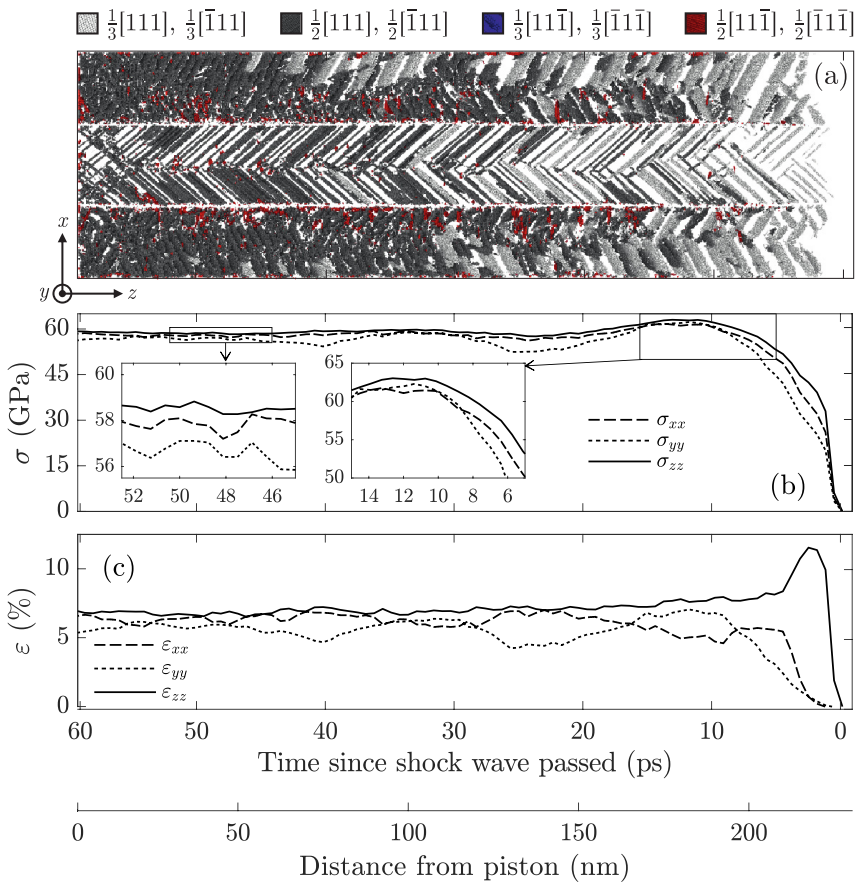

FIG. 15. Visualization of the plasticity mechanisms active in polycrystalline Ta with the HC geometry shocked to $60 \mathrm{GPa}$ along [011] (the positive $z$ direction), alongside profiles of the stresses and elastic strains. (a)-(c) are plotted on the same scale. The $z$ coordinate is primarily expressed in terms of the local time since the compression front passed; the secondary axis shows the distance from the piston generating the shock. (a) Atoms that have participated in plastic flow events, identified via SVA (as in Fig. 4). Visible are planar deformation twins and slip planes with associated Burgers vectors in the $x z$ plane (light and dark grey, respectively). (b) Stresses along the computational cell axes averaged over the cross-section of the central grain. Left and right insets show the late-time stresses and inversion of the stress state, respectively. (c) Elastic strains in the central grain.

with habit planes $(\overline{2} 1 \overline{1})$ and $(21 \overline{1})$, are formed when the grains are compressed along their local [011] ] axis by their rapidly expanding neighbors.

We should stress, however, that the propensity of the CB polycrystal to undergo transverse twinning is most likely an artefact of its high symmetry. HC polycrystals shocked to a similar pressure do not exhibit profuse twinning of this sort, despite the fact that the average stress conditions present in the wake of the shock are comparable (i.e., $\Delta \sigma$ approaches $-2 \mathrm{GPa}$ ). The absence of transverse twins in the HC geometry is demonstrated in Fig. 15. It seems that the comparatively low symmetry of this latter geometry gives rise to a delocalized transverse shear stress field whose magnitude is nowhere sufficient to trigger transverse twinning. By contrast, the highly contrived arrangement of grains in the CB polycrystal is such that, when the grains overshoot the mechanically stable state and enter an inverted stress state, shear stress is strongly concentrated at the grain center. So intense is the concentration, in fact, that at shock pressures exceeding $60 \mathrm{GPa}$ the transverse twinning becomes catastrophic: the closely spaced twins actually coalesce, and form a new, rhombic grain bounded by planes of the type $(\overline{2} 1 \overline{1})$ and $(21 \overline{1})$. In a real polycrystalline target, then, transverse deformation twinning would likely be confined to small regions whose grain configuration locally resembles that of the $\mathrm{CB}$ geometry.

In summary, we find that polycrystals with the $\mathrm{CB}$ geometry undergo deformation twinning on the $(\overline{2} 1 \overline{1})$ and $(21 \overline{1})$ planes when shock-loaded above $60 \mathrm{GPa}$. Deformation of this kind is caused by highly concentrated, inverted transverse shear stresses being generated at the center of each grain by rapid expansion of its neighbors along their local [100] axis. The HC geometry, meanwhile, is not conducive to transverse twinning due to its comparatively low symmetry.

\section{Equilibrium mechanical state}

We saw in Sec. III B that when grains are allowed to expand and contract elastically in directions normal to the loading axis, they can reach a state of lower transverse stress anisotropy $\Delta \sigma$ than laterally confined grains loaded above their HEL. Specifically, $\Delta \sigma$ was limited to at least $1.0 \mathrm{GPa}$ by material strength in the single crystals, while $\sigma_{x x}$ and $\sigma_{y y}$ could converge to within $0.4 \mathrm{GPa}$ for the elastically loaded polycrystals with the CB geometry. In the section that follows, we will determine whether the relief of $\Delta \sigma$ by elastic deformation is still efficient in the plastic regime, and how the efficiency differs between the $\mathrm{CB}$ and $\mathrm{HC}$ geometries. We will also consider the transverse strain anisotropy $\Delta \varepsilon=$ $\varepsilon_{x x}-\varepsilon_{y y}$, which is crucial if one is to make connections with experiment, because the difference (or lack thereof) between elastic strain components can in principle be extracted directly from diffraction data.

The steady-state stresses and elastic strains were obtained by averaging over the portion of the central grain nearest the piston within which the stress and strain profiles were approximately uniform. At low pressures, the profiles suffer some additional nonuniformity due to the grains continuing to execute small SHM-like oscillations even after plastic flow has ceased. The crystals are sufficiently long, though, that the average is taken over at least five oscillation periods, so the additional variation in $\sigma$ and $\varepsilon$ stemming from the SHM averages to a negligible value. At higher pressures, we deliberately truncated the sampling region so as to avoid the region of the crystal nearer the shock front that has undergone transverse twinning, which we are treating as an artefact.

The steady-state transverse stress and strain anisotropies $\Delta \sigma$ and $\Delta \varepsilon$ are shown in Fig. 16 for both the single-crystal control group and polycrystals with the $\mathrm{CB}$ and $\mathrm{HC}$ geometries over the pressure range 40 to $100 \mathrm{GPa}$. In all instances, grains in the $\mathrm{CB}$ polycrystal exhibit lower transverse stress anisotropy than the laterally confined grains do. The elastic relaxation mechanism described in Sec. IIIB is therefore still able to relieve transverse shear stress to a greater extent than transverse slip in the plastic regime, at least for the CB geometry. However, it is clear that the efficiency of elastic relaxation is much reduced above the HEL: most of the data points taken from the CB geometry lie only slightly below the 1.0-GPa single-crystal limit, and only one of them approaches the limiting value of $0.4 \mathrm{GPa}$ reached under elastic loading conditions.

The reduction in efficiency is even more conspicuous in the $\mathrm{HC}$ geometry: overall, $\Delta \sigma$ is no lower in the HC polycrystals 


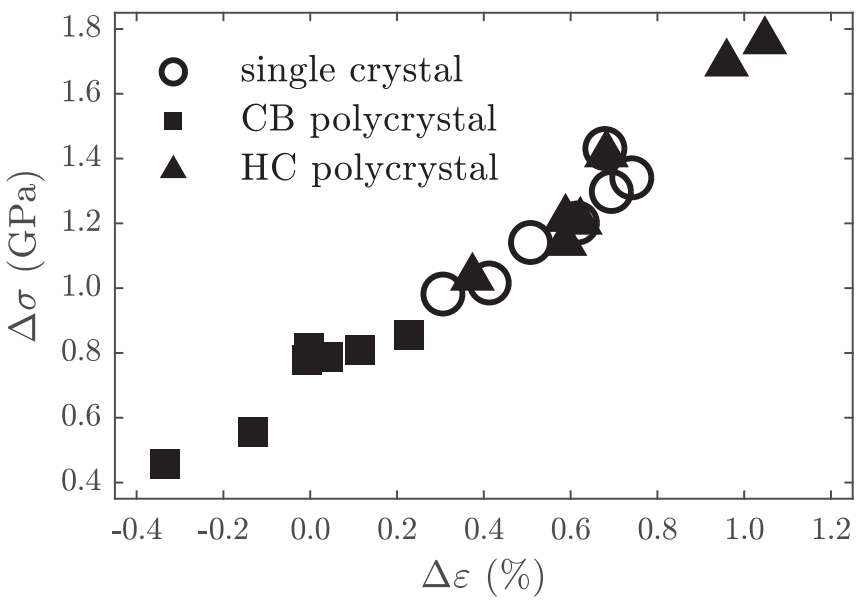

FIG. 16. Steady-state transverse stress and strain anisotropies for single crystals and for the central grain of $\mathrm{CB}$ and $\mathrm{HC}$ polycrystals shocked to between 40 and $100 \mathrm{GPa}$.

than in the single-crystal control group, and is in fact markedly higher in two instances. There were indications in Sec. III B that the cooperative relaxation mechanism is less effective in the $\mathrm{HC}$ than in the CB geometry: Fig. 9 showed that, below the HEL, the HC geometry can tolerate relatively large stress gradients due to the countervailing tractions provided by the off-diagonal shear stress $\sigma_{x y}$. In Fig. 17, we show analogous plots of the off-diagonal stress field for $\mathrm{CB}$ and $\mathrm{HC}$ polycrystals shocked to $60 \mathrm{GPa}$ (i.e., above their HEL). The plots were obtained by calculating $\sigma_{x y}(x, y)$ for notionally relaxed 50 -nm-thick cross-sections of the polycrystals every $0.1 \mathrm{ps}$ for $2.5 \mathrm{ps}$, and then taking the time average- the averaging process somewhat mitigates the considerable noise caused by the thermal fluctuations and spatial inhomogeneities that manifest above the HEL. Once again, we see that shear stress relief is frustrated to a greater degree in the $\mathrm{HC}$ geometry owing to the presence of a comparatively intense off-diagonal stress field. It is perhaps surprising, though, that the cooperative relaxation mechanism is apparently completely ineffectual above the HEL. It is possible that the transverse flow stress of the $\mathrm{HC}$ polycrystals is being enhanced by the presence of their grain boundaries (per the Hall-Petch effect $[80,81]$ ) to such an extent that the cooperative relaxation mechanism is effectively nullified. Further simulations, perhaps of polycrystals with a range of grain sizes, would be necessary to verify this proposition.

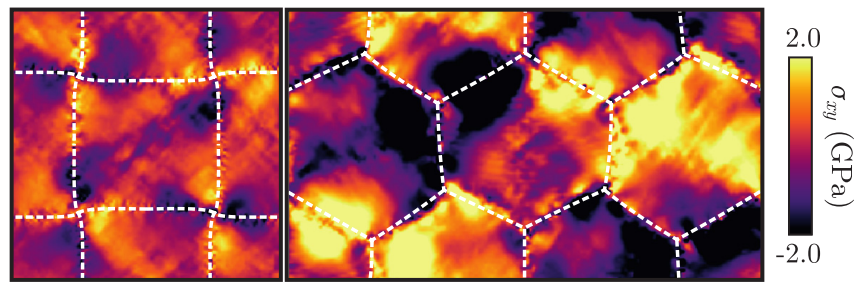

FIG. 17. Comparison of the off-diagonal stress field $\sigma_{x y}$ for $\mathrm{CB}$ (left) and $\mathrm{HC}$ (right) polycrystals shocked to $60 \mathrm{GPa}$ along $z$ following relaxation of the induced stress gradients. Dashed white lines indicate the approximate locations of the grain boundaries after shock compression.
The strain anisotropy $\Delta \varepsilon$, meanwhile, follows much the same pattern as $\Delta \sigma$ : as the difference between $\sigma_{x x}$ and $\sigma_{y y}$ decreases, so too does that between $\varepsilon_{x x}$ and $\varepsilon_{y y}$. In fact, when $\Delta \sigma$ drops below $0.8 \mathrm{GPa}$ (which is only possible in the $\mathrm{CB}$ geometry), the difference between the transverse elastic strains actually changes sign. This conspicuous signature of grain interactions could, given an elastic strain extraction technique of sufficient precision, be verified experimentally, assuming the polycrystal in question is well-represented by the CB geometry; conversely, no such signature would exist if the $\mathrm{HC}$ geometry were the better representation. Whether or not grain interactions could be observed experimentally in this manner therefore depends intimately on the manner in which the grains in the target are oriented and coordinated.

To conclude, we find that when a laterally confined single crystal and a CB polycrystal are shock-loaded to between 40 and $100 \mathrm{GPa}$, the grains in the polycrystal always reach a state of lower transverse stress anisotropy, which is reflected in their elastic strain state. By contrast, the stress state of grains in the $\mathrm{HC}$ geometry is not significantly different from the state they would assume if they were laterally confined. The efficiency of the elastic relaxation mechanism is thus dependent on the geometry of the polycrystal.

\section{DISCUSSION}

The analysis above has demonstrated that the behavior of crystallites in a fiber-textured nanocrystal differs profoundly from that of its single-crystal analogues under shock compression. The salient difference between the nanocrystalline and monocrystalline samples, which was discussed in Sec. III B and will be reiterated here, is one of kinematic freedom. An ideal single crystal is translationally symmetric in directions normal to the loading axis. Thus the global constraint of lateral confinement to which it is subjected during uniaxial shock compression must also apply locally; the crystal is everywhere laterally confined. It is for this reason that any change in the monocrystal's transverse elastic strain state must be realized via plastic flow. When one moves to an aggregate, translational symmetry is broken, and so lateral confinement need not be respected locally. This permits the grains in the aggregate to respond to shear stresses elastically via interactions with their neighbors, which gives rise to the novel effects described in Sec. IIIC, namely the absence of transverse slip at low pressures, the presence of transverse twinning at high pressures, and relaxation of stress anisotropy in the plane normal to the shock.

Clearly, the predictions of this study should be treated with some caution. In an effort to render them amenable to simple analysis and to make as clear as possible their grain interactions, the elementary polycrystals employed in this study were deliberately constructed with highly idealized morphologies and textures. The price of this simplification is the introduction of a number of artificial behaviors, including coherent oscillation of the grains, and catastrophic transverse twinning in the $\mathrm{CB}$ geometry above $60 \mathrm{GPa}$. We also note that the $\mathrm{CB}$ geometry likely exaggerates the extent of the lateral relaxation permitted by grain interactions, because its grains are arranged in such a way that the degree to which the relaxation of one grain frustrates that of its neighbors is 
minimal. It is not immediately clear, then, that the closure of the gap between the two transverse strains seen in the CB geometry would be observed in a real polycrystal-one might expect a priori that the HC geometry would provide a more accurate description of a typical target, in which case interactions would make no perceptible difference to the strain anisotropy. Whatever the case, one should anticipate that interaction effects in a real target will be subtler than our CB polycrystals would suggest.

Nevertheless, it is possible to draw a number of qualitative conclusions from our study whose applicability extends beyond the particular targets considered here. First, the grain interactions that take place under shock conditions are clearly dynamic in nature. Their time-dependence was made apparent in Sec. III B, in which it was shown that the timescale over which neighboring crystallites interact scaled with the transverse grain size $L$ due to the finite propagation speed of stress waves generated at the grain boundaries. We further showed that the transverse twinning observed at higher shock pressures was directly caused by the inertial forces exerted on each grain by its rapidly expanding neighbors. We have also shown that grain interactions can radically alter the crystal's stress state. The typical size of the changes to the transverse stresses caused by interactions is comparable to the strength of the stress gradients produced over the grain boundaries by the uniaxial compression, which in the shock regime is frequently of order gigapascals. Since the stress state governs the RSS acting on any given slip or twinning system, grain interactions also influence which of these systems are active, and hence control the dislocation content of the crystal during shock compression. That grain interactions are able to partially govern stress, strain, and defect evolution means they are worthy of inclusion in any predictive model that seeks to recreate material behavior to better than first order, and they certainly warrant further study in their own right.

There are a number of routes for further work that would shed further light on the physics of grain interactions under shock-loading conditions, and bring us closer to detecting such interactions in experiment. We first suggest that a systematic study of polycrystals with a wider range of morphological and crystallographic textures (rather than just the two presented here) would be highly informative. Our simulations indicate that the efficiency of the cooperative relaxation mode is dictated by the configuration of the grains. A deeper understanding of how each grain frustrates the relaxation of its neighbors might make it possible to predict the efficiency of elastic deformation in a real polycrystal with known texture. We next note that there almost certainly exists a type of target in which grain interaction effects are more conspicuous than they are in our [011] Ta samples, and would therefore have a stronger experimental signature. An exploration of the "parameter space" of targets would therefore be profitable. To narrow the search for a target in which grain interactions are particularly strong, one should first identify desirable material properties, which might include: high material strength (as this would make elastic relaxation effects more conspicuous); a Zener ratio far from unity (which would ensure large stress gradients are induced over the grain boundaries upon shock compression); and a strong texture (as this allows neighboring grains to deform cooperatively with one another, much as the CB polycrystals here do). Finally, it would be instructive to identify and quantify the XRD signatures of grain interaction effects. With a detailed model of how the structure and position of the diffraction peaks changes due to interactions between grains, one could set about identifying the optimal experimental setup in which sensitivity to such interactions is maximized. Studies of this kind could thus inform the design of experiments, and facilitate the direct, in situ observation of grain interactions under shock conditions.

\section{CONCLUSION}

We have performed multi-million atom molecular dynamics simulations of mono- and nanocrystalline tantalum shockcompressed to between 40 and $100 \mathrm{GPa}$, and by comparing the two have identified a number of physical effects that may be ascribed to interactions between neighboring grains. We find that adjacent grains undergo cooperative elastic deformation normal to the shock due to the gigapascal-scale stresses they exert on one another, and are thus able to reach a state of reduced shear stress and strain that would otherwise be made inaccessible by material strength. We further find that the stress changes precipitated by grain interactions are able to activate and deactivate conventional plasticity mechanisms depending on the shock pressure, and, as a corollary to this, have some degree of control over the dislocation content of the crystal during compression. In brief, our analysis has shown that the pervasive influence of grain interactions under shock-loading conditions must not be underestimated, and surely deserves further computational study.

\section{ACKNOWLEDGMENTS}

The authors would like to thank R. E. Rudd for useful discussions. P. G. H., D. M., and J. S. W. are grateful to AWE for support via the Oxford center for High Energy Density Science (OxCHEDS).
[1] This assertion is true provided the boundaries between the grains do not fail.

[2] R. Becker, Analysis of texture evolution in channel die compression-i. effects of grain interaction, Acta Metall. Mater. 39, 1211 (1991).

[3] S. R. Kalidindi, C. A. Bronkhorst, and L. Anand, Crystallographic texture evolution in bulk deformation processing of fcc metals, J. Mech. Phys. Solids 40, 537 (1992).
[4] R. Becker and S. Panchanadeeswaran, Effects of grain interactions on deformation and local texture in polycrystals, Acta Metall. Mater. 43, 2701 (1995).

[5] A. J. Beaudoin, P. R. Dawson, K. K. Mathur, and U. F. Kocks, A hybrid finite element formulation for polycrystal plasticity with consideration of macrostructural and microstructural linking, Int. J. Plasticity 11, 501 (1995). 
[6] S. Panchanadeeswaran, R. D. Doherty, and R. Becker, Direct observation of orientation change by channel die compression of polycrystalline aluminum - use of a split sample, Acta Mater. 44, 1233 (1996).

[7] G. B. Sarma, B. Radhakrishnan, and T. Zacharia, Finite element simulations of cold deformation at the mesoscale, Comput. Mater. Sci. 12, 105 (1998).

[8] F. Delaire, J. L. Raphanel, and C. Rey, Plastic heterogeneities of a copper multicrystal deformed in uniaxial tension: experimental study and finite element simulations, Acta Mater. 48, 1075 (2000).

[9] A. Bhattacharyya, E. El-Danaf, S. R. Kalidindi, and R. D. Doherty, Evolution of grain-scale microstructure during large strain simple compression of polycrystalline aluminum with quasi-columnar grains: Oim measurements and numerical simulations, Int. J. Plasticity 17, 861 (2001).

[10] H. Toda, T. Kamiko, Y. Tanabe, M. Kobayashi, D. J. Leclere, K. Uesugi, A. Takeuchi, and K. Hirayama, Diffractionamalgamated grain boundary tracking for mapping $3 \mathrm{~d}$ crystallographic orientation and strain fields during plastic deformation, Acta Mater. 107, 310 (2016).

[11] G. B. Sarma and P. R. Dawson, Effects of interactions among crystals on the inhomogeneous deformations of polycrystals, Acta Mater. 44, 1937 (1996).

[12] D. P. Mika and P. R. Dawson, Effects of grain interaction on deformation in polycrystals, Mater. Sci. Eng. A 257, 62 (1998).

[13] F. Barbe, S. Forest, and G. Cailletaud, Intergranular and intragranular behavior of polycrystalline aggregates.part 2: Results, Int. J. Plasticity 17, 537 (2001).

[14] U. Lienert, T.-S. Han, J. Almer, P. R. Dawson, T. Leffers, L. Margulies, S. F. Nielsen, H. F. Poulsen, and S. Schmidt, Investigating the effect of grain interaction during plastic deformation of copper, Acta Mater. 52, 4461 (2004).

[15] M. F. Ashby, The deformation of plastically non-homogeneous materials, Philos. Mag. 21, 399 (1970).

[16] L. P. Evers, D. M. Parks, W. A. M. Brekelmans, and M. G. D. Geers, Crystal plasticity model with enhanced hardening by geometrically necessary dislocation accumulation, J. Mech. Phys. Solids 50, 2403 (2002).

[17] T. Ohashi, R. I. Barabash, J. W. L. Pang, G. E. Ice, and O. M. Barabash, X-ray microdiffraction and strain gradient crystal plasticity studies of geometrically necessary dislocations near a ni bicrystal grain boundary, Int. J. Plasticity 25, 920 (2009).

[18] P. D. Littlewood, T. B. Britton, and A. J. Wilkinson, Geometrically necessary dislocation density distributions in ti-6al-4v deformed in tension, Acta Mater. 59, 6489 (2011).

[19] J. W. Hutchinson and R. Hill, Elastic-plastic behavior of polycrystalline metals and composites, Proc. R. Soc. London A 319, 247 (1970).

[20] S. V. Harren and R. J. Asaro, Nonuniform deformations in polycrystals and aspects of the validity of the taylor model, J. Mech. Phys. Solids 37, 191 (1989).

[21] M. J Suggit, A. Higginbotham, J. A. Hawreliak, G. Mogni, G. Kimminau, P. Dunne, A. J. Comley, N. Park, B. A. Remington, and J. S. Wark, Nanosecond white-light Laue diffraction measurements of dislocation microstructure in shock-compressed single-crystal copper, Nat. Commun. 3, 1224 (2012).

[22] C. E. Wehrenberg, D. McGonegle, C. Bolme, A. Higginbotham, A. Lazicki, H. J. Lee, B. Nagler, H.-S. Park, B. A. Remington, R. E. Rudd, M. Sliwa, M. Suggit, D. Swift, F. Tavella,
L. Zepeda-Ruiz, and J. S. Wark, In situ X-ray diffraction measurement of shock-wave-driven twinning and lattice dynamics, Nature (London) 550, 496 (2017).

[23] W. J. Murphy, A. Higginbotham, G. Kimminau, B. Barbrel, E. M. Bringa, J. Hawreliak, R. Kodama, M. Koenig, W. McBarron, M. A. Meyers, B. Nagler, N. Ozaki, N. Park, B. Remington, S. Rothman, S. M. Vinko, T. Whitcher, and J. S. Wark, The strength of single crystal copper under uniaxial shock compression at $100 \mathrm{GPa}$, J. Phys.: Condens. Matter 22, 065404 (2010).

[24] D. Milathianaki, S. Boutet, G. J. Williams, A. Higginbotham, D. Ratner, A. E. Gleason, M. Messerschmidt, M. M Seibert, D. C. Swift, P. Hering, J. Robinson, W. E. White, and J. S. Wark, Femtosecond visualization of lattice dynamics in shock-compressed matter, Science 342, 220 (2013).

[25] A. J. Comley, B. R. Maddox, R. E. Rudd, S. T. Prisbrey, J. A. Hawreliak, D. A. Orlikowski, S. C. Peterson, J. H. Satcher, A. J. Elsholz, H.-S. Park, B. A. Remington, N. Bazin, J. M. Foster, P. Graham, N. Park, P. A. Rosen, S. R. Rothman, A. Higginbotham, M. Suggit, and J. S. Wark, Strength of ShockLoaded Single-Crystal Tantalum [100] Determined Using in Situ Broadband X-Ray Laue Diffraction, Phys. Rev. Lett. 110, 115501 (2013).

[26] C. E. Wehrenberg, A. J. Comley, N. R. Barton, F. Coppari, D. Fratanduono, C. M. Huntington, B. R. Maddox, H.-S. Park, C. Plechaty, S. T. Prisbrey, B. A. Remington, and R. E. Rudd, Lattice-level observation of the elastic-to-plastic relaxation process with subnanosecond resolution in shock-compressed ta using time-resolved in situ laue diffraction, Phys. Rev. B 92, 104305 (2015).

[27] M. Sliwa, D. McGonegle, C. Wehrenberg, C. A. Bolme, P. G. Heighway, A. Higginbotham, A. Lazicki, H. J. Lee, B. Nagler, H. S. Park, R. E. Rudd, M. J. Suggit, D. Swift, F. Tavella, L. Zepeda-Ruiz, B. A. Remington, and J. S. Wark, Femtosecond X-Ray Diffraction Studies of the Reversal of the Microstructural Effects of Plastic Deformation During Shock Release of Tantalum, Phys. Rev. Lett. 120, 265502 (2018).

[28] G. I. Taylor, Plastic strain in metals, J. Inst. Metals 62, 307 (1937).

[29] J. F. W. Bishop and R. Hill, Xlvi. a theory of the plastic distortion of a polycrystalline aggregate under combined stresses. Lond. Edinb. Dubl. Phil. Mag. 42, 414 (1951).

[30] H. Honneff and H. Mecking, A method for the determination of the active slip systems and orientation changes during single crystal deformation, in Proc. 5th Int. Conf. on Texture of Materials, edited by G. Gottstein and K. Lucke (Springer, 1978), pp. 265-275.

[31] U. F. Kocks and G. R. Canova, in Deformation of Polycrystals, edited by N. Hansen (Ris $\varnothing$ National Laboratory, 1980), p. 35.

[32] Here, we use the term Taylor-type to refer to any model underpinned by an assumption of strain homogeneity. This includes Taylor's original prescription, in which every strain component is constant throughout the aggregate, and variations thereof such as the relaxed constraints approach, where select strain components are allowed to vary.

[33] E. Kröner, Zur plastischen verformung des vielkristalls, Acta Metall. 9, 155 (1961).

[34] B. Budiansky and T. T. Wu, Theoretical prediction of plastic strains of polycrystals, in Proc. 4th U.S. Natn. Congr. Appl. 
Mech. (American Society of Mechanical Engineers, 1962), p. 1175 .

[35] R. Hill, A self-consistent mechanics of composite materials, J. Mech. Phys. Solids 13, 213 (1965).

[36] F. Roters, P. Eisenlohr, T. Bieler, and D. Raabe, Crystal Plasticity Finite Element Methods (Wiley-VCH, Weinheim, Germany, 2010).

[37] Note that it is possible for single crystals to fragment, provided their orientation relative to the loading axes is such that two or more slip systems suffer similar resolved shear stresses.

[38] K. Kadau, T. C. Germann, P. S. Lomdahl, R. C. Albers, J. S. Wark, A. Higginbotham, and B. L. Holian, Shock Waves in Polycrystalline Iron, Phys. Rev. Lett. 98, 135701 (2007).

[39] N. Park, Modelling Shocks using Molecular Dynamics, Ph.D. thesis, Cranfield University, 2009.

[40] S.-N. Luo, T. Germann, D. L. Tonks, and Q. An, Shock wave loading and spallation of copper bicrystals with asymmetric $\Sigma 3\langle 110\rangle$ tilt grain boundaries, J. Appl. Phys. 108, 093526 (2010).

[41] H. Pham, B. Arman, S.-N. Luo, and T. Cagin, Shock compression and spallation of palladium bicrystals with a $\Sigma 5$ grain boundary, J. Appl. Phys. 109, 086107 (2011).

[42] E. Q. Lin, H. J. Shi, L. S. Niu, and E. Z. Jin, Shock response of copper bicrystals with a $\Sigma 3$ asymmetric tilt grain boundary, Comput. Mater. Sci. 59, 94 (2012).

[43] W. Z. Han, Q. An, S. N. Luo, T. C. Germann, D. L. Tonks, and W. A. Goddard, III, Deformation and spallation of shocked cu bicrystals with $\Sigma 3$ coherent and symmetric incoherent twin boundaries, Phys. Rev. B 85, 024107 (2012).

[44] N. Gunkelmann, E. M. Bringa, D. R. Tramontina, C. J. Ruestes, M. J. Suggit, A. Higginbotham, J. S. Wark, and H. M. Urbassek, Shock waves in polycrystalline iron: Plasticity and phase transitions, Phys. Rev. B 89, 140102 (2014).

[45] L. Wang, J. E, Y. Cai, F. Zhao, D. Fan, and S.-N. Luo, Shockinduced deformation of nanocrystalline al: Characterization with orientation mapping and selected area electron diffraction, J. Appl. Phys. 117, 084301 (2015).

[46] M. X. Tang, J. C. E, L. Wang, and S. N. Luo, Loading-path dependent deformation of nanocrystalline ta under single- and double-shock, and quasi-isentropic compression, J. Appl. Phys. 121, 115901 (2017).

[47] A. Stukowski, Visualization and analysis of atomistic simulation data with OVITO-the open visualization tool, Model. Simul. Mater. Sci. Eng. 18, 015012 (2009).

[48] S. Plimpton, Fast parallel algorithms for short-range molecular dynamics, J. Comput. Phys. 117, 1 (1995).

[49] R. Ravelo, T. C. Germann, O. Guerrero, Q. An, and B. L. Holian, Shock-induced plasticity in tantalum single crystals: Interatomic potentials and large-scale molecular-dynamics simulations, Phys. Rev. B 88, 134101 (2013).

[50] H. Cynn and C.-S. Yoo, Equation of state of tantalum to 174 gpa, Phys. Rev. B 59, 8526 (1999).

[51] A. Dewaele, P. Loubeyre, and M. Mezouar, Equations of state of six metals above 94 gpa, Phys. Rev. B 70, 094112 (2004).

[52] A. C. Mitchell and W. J. Nellis, Shock compression of aluminium, copper, and tantalum, J. Appl. Phys. 52, 3363 (1981).

[53] A. Dewaele, M. Mezouar, N. Guignot, and P. Loubeyre, High Melting Points of Tantalum in a Laser-Heated Diamond Anvil Cell, Phys. Rev. Lett. 104, 255701 (2010).
[54] T. Schneider and E. Stoll, Molecular-dynamics study of a three-dimensional one-component model for distortive phase transitions, Phys. Rev. B 17, 1302 (1978).

[55] J. A. Zimmerman, C. L. Kelchner, P. A. Klein, J. C. Hamilton, and S. M. Foiles, Surface Step Effects on Nanoindentation, Phys. Rev. Lett. 87, 165507 (2001).

[56] R. E. Rudd, High-rate plastic deformation of nanocrystalline tantalum to large strains: Molecular dynamics simulation, Mater. Sci. Forum 633-634, 3 (2009).

[57] A. Higginbotham, M. J. Suggit, E. M. Bringa, P. Erhart, J. A. Hawreliak, G. Mogni, N. Park, B. A. Remington, and J. S. Wark, Molecular dynamics simulations of shock-induced deformation twinning of a body-centered-cubic metal, Phys. Rev. B 88, 104105 (2013).

[58] D. McGonegle, D. Milathianaki, B. A. Remington, J. S. Wark, and A. Higginbotham, Simulations of in situ X-ray diffraction from uniaxially compressed highly textured polycrystalline targets, J. Appl. Phys. 118, 065902 (2015).

[59] J. D. Honeycutt and H. C. Andersen, Molecular dynamics study of melting and freezing of small lennard-jones clusters, J. Phys. Chem. 91, 4950 (1987).

[60] A. Stukowski, Structure identification methods for atomistic simulations of crystalline materials, Model. Simul. Mater. Sci. Eng. 20, 045021 (2012).

[61] E. A. Lazar, J. Han, and D. J. Srolovitz, A topological framework for local structure analysis of condensed matter, Proc. Natl. Acad. Sci. USA 112, E5769 (2015).

[62] A. Stukowski and K. Albe, Extracting dislocations and nondislocation crystal defects from atomistic simulation data, Model. Simul. Mater. Sci. Eng. 18, 085001 (2010).

[63] A. Stukowski, A triangulation-based method to identify dislocations in atomistic models, J. Mech. Phys. Solids 70, 314 (2014).

[64] A. Stukowski, V. V. Bulatov, and A. Arsenlis, Automated identification and indexing of dislocations in crystal interfaces, Model. Simul. Mater. Sci. Eng. 20, 085007 (2012).

[65] D. Tramontina, C. Ruestes, Y. Tang, and E. Bringa, Orientationdependent response of defective tantalum single crystals, Comput. Mater. Sci. 90, 82 (2014).

[66] C.-H. Lu, E. N. Hahn, B. A. Remington, B. R. Maddox, E. M. Bringa, and M. A. Meyers, Phase transformation in tantalum under extreme laser deformation, Sci. Rep. 5, 15064 (2015).

[67] B. Pang, S. Case, I. P. Jones, J. C. F. Millett, G. Whiteman, Y. L. Chiu, and C. A. Bronkhorst, The defect evolution in shock loaded tantalum single crystals, Acta Mater. 148, 482 (2018).

[68] J. Florando, N. Barton, B. S. El-Dasher, J. Mcnaney, and M. Kumar, Analysis of deformation twinning in tantalum single crystals under shock loading conditions, J. Appl. Phys. 113, 083522 (2013).

[69] C. Ruestes, A. Stukowski, Y. Tang, D. Tramontina, P. Erhart, B. Remington, H. Urbassek, M. Meyers, and E. Bringa, Atomistic simulation of tantalum nanoindentation: Effects of indenter diameter, penetration velocity, and interatomic potentials on defect mechanisms and evolution, Mater. Sci. Eng. A 613, 390 (2014).

[70] One can show by rotating the stiffness tensor into a frame in which [100], [011] , and [011] are aligned with the computational cell axes that, in a state of uniaxial strain along [011] by $\varepsilon_{z z}$, the stresses are given by $\sigma_{x x}=c_{12} \varepsilon_{z z}, \sigma_{y y}=\left[\left(c_{11}+c_{12}\right) / 2-c_{44}\right] \varepsilon_{z z}$, and $\sigma_{z z}=\left[\left(c_{11}+c_{12}\right) / 2+c_{44}\right] \varepsilon_{z z}$, where the $\left\{c_{i j}\right\}$ are the cubic 
elastic constants. If the ambient lattice constants for tantalum are used $\left(c_{11}=263 \mathrm{GPa}, c_{12}=161 \mathrm{GPa}, c_{44}=82 \mathrm{GPa}\right)$, we correctly predict $\sigma_{z z}>\sigma_{x x}>\sigma_{y y}$.

[71] W. Voigt, Ueber die beziehung zwischen den beiden elasticitätsconstanten isotroper körper, Ann. Phys. 274, 573 (1889).

[72] A. Reuss, Berechnung der fließgrenze von mischkristallen auf grund der plastizitätsbedingung für einkristalle. Z. Angew. Math. Mech. 9, 49 (1929).

[73] D. R. Lide, CRC Handbook of Chemistry and Physics, 84th ed. (CRC Press, Florida, USA, 2003-2004).

[74] V. Dremov, A. Rykounov, F. A. Sapozhnikov, A. Karavaev, S. V. Yakovlev, G. V. Ionov, and M. V. Ryzhkov, Cold melting of beryllium: Atomistic view on z-machine experiments, J. Appl. Phys. 118, 035901 (2015).

[75] R. F. Smith, J. H. Eggert, R. Jeanloz, T. S. Duffy, D. G. Braun, J. R. Patterson, R. E. Rudd, J. Biener, A. E. Lazicki, A. V. Hamza, J. Wang, T. Braun, L. X. Benedict, P. M. Celliers, and G. W. Collins, Ramp compression of diamond to five terapascals, Nature (London) 511, 330 (2014).

[76] G. I. Taylor, The deformation of crystals of $\beta$-brass, Proc. R. Soc. London A 118, 1 (1928).

[77] G. Sainath and B. K. Choudhary, Orientation dependent deformation behavior of bcc iron nanowires, Comput. Mater. Sci. 111, 406 (2016).

[78] J.-Y. Kim, D. Jang, and J. R. Greer, Crystallographic orientation and size dependence of tension-compression asymmetry in molybdenum nano-pillars, Int. J. Plasticity 28, 46 (2012).

[79] D. K. Bowen, J. W. Christian, and G. Taylor, Deformation properties of niobium single crystals, Can. J. Phys. 45, 903 (1967).

[80] E. O. Hall, The Deformation and Ageing of Mild Steel: III Discussion of Results, Proc. Phys. Soc. London B 64, 747 (1951).

[81] N. J. Petch, The cleavage strength of polycrystals, J. Iron Steel Inst. 174, 25 (1953). 